\title{
Beyond good and evil: A putative continuum-sorting hypothesis for the functional role of proBDNF/BDNF-propeptide/mBDNF in antidepressant treatment
}

\author{
Cassiano R.A.F. Diniz ${ }^{\mathrm{a}, \mathrm{c}, *}$, Plinio C Casarotto ${ }^{\mathrm{a}, \mathrm{b}}$, Leonardo Resstel ${ }^{\mathrm{a}}$, Sâmia R.L. Joca ${ }^{\mathrm{a}, \mathrm{c}, \mathrm{d}}$ \\ a School of Medicine, Campus USP, Ribeirão Preto, SP 14049-900, Brazil \\ ${ }^{\mathbf{b}}$ Neuroscience Center - HILife, University of Helsinki, Finland \\ ${ }^{\mathrm{c}}$ Department of Physics and Chemistry, School of Pharmaceutical Sciences of Ribeirão Preto, Campus USP, Ribeirão Preto, SP 14040-904, Brazil \\ d Translational Neuropsychiatry Unit, Department of Clinical Medicine, Aarhus University, Denmark
}

\section{A R T I C L E I N F O}

\section{Keywords:}

BDNF

proBDNF/BDNF-propeptide

Stress

Continuum-sorting hypothesis

Depression

PTSD

\begin{abstract}
A B S T R A C T
Depression and posttraumatic stress disorder are assumed to be maladaptive responses to stress and antidepressants are thought to counteract such responses by increasing BDNF (brain-derived neurotrophic factor) levels. BDNF acts through TrkB (tropomyosin-related receptor kinase B) and plays a central role in neuroplasticity. In contrast, both precursor proBDNF and BDNF propeptide (another metabolic product from proBDNF cleavage) have a high affinity to p75 receptor (p75R) and usually convey apoptosis and neuronal shrinkage. Although BDNF and proBDNF/propeptide apparently act in opposite ways, neuronal turnover and remodeling might be a final common way that both act to promote more effective neuronal networking, avoiding neuronal redundancy and the misleading effects of environmental contingencies. This review aims to provide a brief overview about the BDNF functional role in antidepressant action and about p75R and TrkB signaling to introduce the "continuum-sorting hypothesis." The resulting hypothesis suggests that both BDNF/proBDNF and $\mathrm{BDNF}$ /propeptide act as protagonists to fine-tune antidepressant-dependent neuroplasticity in crucial brain structures to modulate behavioral responses to stress.
\end{abstract}

\section{Introduction}

Antidepressants are widely used to treat several psychiatric and neurologic disorders, such as depression, anxiety, posttraumatic stress disorder (PTSD), obsessive-compulsive disorder, and neuropathic pain. Major classes of antidepressant drugs include selective serotonin reuptake inhibitors (SSRI) and non-selective serotonin/noradrenaline reuptake inhibitors (SNRI). Both classes increase the available monoamine levels in the synaptic cleft, thus implicating this mechanism in the neurobiology of such disorders. In general, the efficacy of antidepressants is limited. Antidepressant drugs also present adverse undesirable effects whose manifestation impair treatment adherence. Thus, the study of antidepressant mechanisms of action, as well as the neurobiology of psychiatry disorders, is essential to search new pharmacological tools that promote better efficacy and adherence to the treatment. Although the primary antidepressant mechanism of action is related to an increase of monoaminergic availability, different classes of such compounds are also able to increase the mature brain-derived neurotrophic factor (mBDNF) levels in the forebrain, after chronic treatment (for review see (Castren and Antila, 2017)). This last effect is suggested to be necessary for antidepressant response and, thus, it suggests mBDNF as a fundamental player to promote the development of new therapeutics. Despite being a widely studied molecule, the role of BDNF's precursor, and its byproduct propeptide, is scarcely understood. As such, the present review intends to briefly overview the basic aspects of mBDNF functional role in antidepressant action and the usual signaling involved either with proBDNF/propeptide or with mBDNF to introduce the main idea named "continuum-sorting hypothesis." This concept will emphasize the signaling of both neurotrophin receptors, p75 (p75R) and TrkB (tropomyosin-related receptor kinase B), as parallel pathways that, in combination with the classical effects of mBDNF itself, promote neuronal turnover and remodeling. The "continuumsorting hypothesis" postulates that antidepressants might regulate the balance of TrkB/p75R signaling on demand to find a balanced way to foment more effective neuronal networking, avoiding neuronal redundancy and the misleading outcome of environmental contingencies. The hypothesis was conceived in response to psychiatric disorders arising from maladaptive behavioral responses to stress, such as

\footnotetext{
* Corresponding author at: School of Medicine of Ribeirão Preto (FMRP) University of São Paulo (USP). Av. Bandeirantes, N 3900, 14049-900, Ribeirão Preto - SP, Brazil.

E-mail address: cassiano.diniz@usp.br (C.R.A.F. Diniz).
} 
depression and posttraumatic stress disorder, but we parsimoniously suggest that the concept might also be expanded to other conditions. Since the caution in distinguishing mBDNF and proBDNF is recent, if the BDNF term is written along the text without a prefix in this review, it means that the original manuscript being referred to has not distinguished between mBDNF or proBDNF, or it has built the discussion around broad concepts. However, with the exception of the neutral aspect concerning the description of gene and transcripts, the most part of the logical construct (where distinction of BDNF's forms were not addressed) makes better sense based on the mBDNF's rationality. Accordingly, the term proBDNF or propeptide has been used whenever necessary and possible.

\section{Antidepressant effects on neuronal plasticity}

Antidepressant drugs have been widely recognized to promote neuronal plasticity, such as survival, dendritic sprouting, synaptogenesis, axonal approach, and increased synaptic strength. For example, fluoxetine (FLX, SSRI)-treated animals exhibited an overall increase in the amplitude of evoked field excitatory postsynaptic potential (fEPSPs) and long-term potentiation (LTP) of fEPSPs in lateral amygdala (Karpova et al., 2011). Increased activity-induced synaptic LTP in hippocampal dentate gyrus (DG) was also described after chronic FLX treatment (Bath et al., 2012; Wang et al., 2008). LTP is suggested as synaptic mechanisms that supports learning and memory, since an increase in the efficiency of synaptic transmissions is verified after highfrequency stimulation of monosynaptic pathways (Bliss and Collingridge, 1993).

Chronic FLX treatment also increases neuronal survival of adult newborn neurons, and enhances dendritic complexity in both amygdala and hippocampus (Wang et al., 2008; Jiang et al., 2014). Another study showed both FLX and imipramine (IMI, SNRI) chronic treatment increased neuronal survival and proliferation in the dentate gyrus of hippocampus (Sairanen et al., 2005). Maya-Vetencourt and coworkers demonstrated that FLX treatment induced ocular dominance shift of visual cortex neurons in favor of the non-deprived eye, as it has also been able to recover visual acuity in adult amblyopic rats (Maya Vetencourt et al., 2008, 2011). Therefore, antidepressant chronic treatment apparently promotes plasticity reactivation in the visual cortex.

In addition, the percentage of perineuronal nets (PNN) neurons expressing parvalbumin decreased in the basolateral amygdala (BLA) and in the hippocampal CA1 field of rats chronically treated with FLX, whereas polysialynated neuronal cell-adhesion molecule (PSA-NCAM) levels increased (Karpova et al., 2011). Accordingly, the number of PSA-NCAM immunoreactive neurons and intensity of PSA-NCAM neuropil immunostaining were both increased in the medial prefrontal cortex (mPFC) of rats chronically treated with FLX (Varea et al., 2007). PSA-NCAM expression is important to structural neuroplasticity, such as axonal growth (Zhang et al., 1992) and synaptic reorganization (Seki and Rutishauser, 1998). FLX treatment also increased levels of hippocampal synaptic protein (O'Leary et al., 2009) and density of dendritic spine in the CA1 and CA3 fields of female rats (Hajszan et al., 2005). In addition, chronic FLX treatment reduced the levels of mature granule cell marker calbindin, induced active somatic membrane properties resembling immature granule cells, and reduced mossy fiber synaptic facilitation to a juvenile-like level (Kobayashi et al., 2010). Altogether, these antidepressant effects are thought to mimic developmental-like plasticity (Castren and Antila, 2017), which might have an effect in how the brain reacts to stress to promote adaptation.

\section{Stress and antidepressant effects on BDNF signaling}

\subsection{BDNF: synthesis, storage and release}

A key role for BDNF has been suggested in the mediation of antidepressant-induced neuroplasticity. BDNF is a member of neurotrophic factor family found mostly in the central nervous system. BDNF is considered to be important for neuroplasticity, such as synaptic remodeling, neuronal differentiation, axonal growth, and neuronal survival (Park and Poo, 2013). Initially, mRNA BDNF transcripts are translated in the endoplasmic reticulum as a precursor protein, proBDNF (30-32 kDa), which may be further cleaved within the Golgi complex by furin or within vesicles by pro-protein convertases ( $\mathrm{Lu}$ et al., 2005; Pang et al., 2004; Lu, 2003a). ProBDNF can also be secreted into the synaptic cleft (Yang et al., 2009), where it can be subsequently cleaved by metalloproteinases and/or plasmin, giving rise to $\mathrm{mBDNF}$ and the protein portion related to the pro-domain, BDNF propeptide (Pang et al., 2004; Lee et al., 2001). To promote the proper folding of the mature domain, the pro-domain must interact with sortilin receptor in the Golgi wall, which in turn allows a motif in the mature domain to connect to carboxypeptidase E, and then BDNF is sorted into large dense core vesicles to be released from the regulated secretory pathway (Lu et al., 2005). Absence or unavailability of the motif in the mature domain sorts BDNF to be released from the constitutive pathway (Lu et al., 2005). Accordingly, a truncated form of sortilin, which lacks the cytoplasmic tail domain, causes BDNF missorting by avoiding BDNF release from the regulated to constitutive secretory pathway (Chen et al., 2005). Trafficking of BDNF to lysosomal degradation also requires the sortilin cytoplasmic tail, thus implying that sortilin shedding favors BDNF availability by impairing lysosomal targeting and its degradation (Evans et al., 2001).

BDNF gene structure has a complex organization and multiple transcripts are possible from the genetic sequence. Recent data indicates mouse and rat BDNF genes consist of one $3^{\prime}$ exon-encoding BDNF protein (exon IX) and eight $5^{\prime}$ noncoding exons, thus each BDNF transcript contains one $5^{\prime}$ exon spliced to the common 3' exon (Aid et al., 2007; West et al., 2014). Twenty-two different mRNAs may be obtained from the BDNF gene, since exon IX mRNA alternatively includes a 5'extended coding exon (exon IXA), that transcript variants from exon II, such as IIA, IIB, and IIC were found and that each transcript may use one of two alternative polyadenylation signals in the 3' exon (giving rise to short and long 3'UTR) (Aid et al., 2007; West et al., 2014). Transcripts with splice variants containing 5 'noncoding exons 1 , 2 , 4, or 6 altogether portray 95\% of the global BDNF mRNA in mice, rats, and human cortex (Aid et al., 2007; Pruunsild et al., 2007). In the human BDNF gene, 9 functional promoters and 11 exons are found, and as in rodents, the 3 'exon encodes the BDNF protein (Pruunsild et al., 2007).

\subsection{BDNF modulation of neuronal plasticity}

BDNF is a high affinity binder for TrkB (Soppet et al., 1991). Phosphorylation of residues Y705/6 at the cytoplasmic autoregulatory loop of the kinase domain (catalytic domain) is considered a critical step in TrkB activation (Huang and Reichardt, 2001), followed by phosphorylation of other tyrosine residues, such as Y515 and Y816 (Huang and Reichardt, 2001). Activation of Y515 residue triggers the MAPK/AKT pathways, while phosphorylation of Y816 induces activation of the phospholipase C gamma (PLC $\gamma$ )-mediated signaling pathway (Rantamaki et al., 2007). BDNF-induced TrkB activation is able to promote survival of cultured embryonic mesencephalic dopaminergic neurons (Hyman et al., 1991; Knusel et al., 1991), cholinergic neurons in the basal forebrain (Alderson et al., 1990), retinal ganglion cells (Johnson et al., 1986), cortical embryonic neurons (Ghosh et al., 1994), among others. in vivo, BDNF was also able to promote survival of neurons of the nodose ganglion and dorsal root ganglion (Hofer and Barde, 1998). Peripheral chronic BDNF administration in mice also enhanced cell survival in hippocampus and PFC (Schmidt and Duman, 2010).

Besides the effects in cell survival and proliferation, mBDNF is able to support late hippocampal LTP through a TrkB-dependent mechanism 
(Pang et al., 2004; Panja et al., 2014; Gooney and Lynch, 2001; Tanaka et al., 2008). Accordingly, BDNF administration in BDNF knockout mice rescues hippocampal LTP deficits at the Schaffer collateral-CA1 synapse (Patterson et al., 1996). Moreover, induction of LTP in the hippocampal dentate gyrus by perforant path stimulation was sufficient to increase BDNF mRNA expression in granular neurons of dentate gyrus (Castren et al., 1993). Resembling BDNF/TrkB-Y816 downstream signaling, LTP hippocampal stimulation also depends on PLC $\gamma$ signaling (Gartner et al., 2006), thus implicating this mechanism in BDNF-induced effects in LTP modulation.

In cultured hippocampal neurons, both gradual and acute BDNF delivery changed neurite growth and spine morphology ( $\mathrm{Ji}$ et al., 2010). Tanaka and coworkers observed that spike-timing protocol, which is a trigger to release BDNF from stimulus-induced LTP, foment a protein synthesis-dependent enlargement in spine volume at the hippocampal CA3-CA1 synapse (Tanaka et al., 2008). In agreement, impairment in synaptic function is observed in BDNF knockout mice, along with a decrease in synaptophysin and synaptobrevin levels. Since BDNF replacement reversed such impairments (Pozzo-Miller et al., 1999), it suggests BDNF modulates synaptic vesicle docking at the active zones of CA1 synapses. Altogether, these data support BDNF and TrkB signaling effects in synaptic efficiency (LTP) and cell morphology (neurite outgrowth, axonal sprouting, and neuronal survival) to promote neuronal plasticity.

\subsection{Stress modulates brain neuroplasticity and BDNF levels}

Repeated or sustained stress exposure usually affects neuroplasticity negatively. For example, chronic stress reduced NCAM levels in the hippocampus, whereas prenatal stress decreased NCAM levels in hippocampus and cortical regions (Sandi, 2004). Accordingly, chronic infusion of corticosterone, which is considered a stress hormone, mimicked the effect of chronic stress exposure by reducing NCAM expression either in frontal cortex (Sandi and Loscertales, 1999) or in DG (Nacher et al., 2004). Since the NCAM role is associated with neurite plasticity (Seki and Rutishauser, 1998), the aforementioned effects might reflect impairments in neurite morphology in response to stress. In fact, ultrastructural synaptic parameters in mossy fiber-CA3 synapse were found altered after 21 days of restraint-stress, including a vesicular rearrangement and an increase at the area of mossy fiber terminal occupied by mitochondria (Magarinos et al., 1997). Furthermore, adult animals stressed at early life showed decrease in the complexity of CA1 dendritic tree and LTP impairment in CA3 field of hippocampal slices (Brunson et al., 2005). Chronic restraint stress also decreased dendritic spine volume and surface area, reduced large spines, and increased small spine numbers in the mPFC (Radley et al., 2008), whereas decreased proliferation and survival of adult newborn hippocampal neurons, reduced granule cell number and layer volume (Pham et al., 2003). Indeed, different chronic stressors, including chronic unpredictable stress, can induce an impairment of hippocampal neurogenesis (Vollmayr et al., 2007). In addition, broad apical dendrite abnormality (dendritic atrophy and spine loss) in pyramidal neurons of PFC areas such as anterior cingulate, prelimbic, and infralimbic is noticed after animal exposure to stress or to glucocorticoids (Izquierdo et al., 2006; Liston et al., 2006; Radley et al., 2006).

Accordingly, stress exposure is also able to negatively impact BDNF levels and neuroplasticity in a supposed entwined way, then decreasing inter-neural connections and global brain network wiring. For instance, $24 \mathrm{~h}$ of maternal deprivation at postnatal day 9 decreased both adult hippocampal BDNF protein and mRNA expression (Roceri et al., 2002). Systemic corticosterone infusion mimicked immobilization stress effect, decreasing BDNF mRNA expression in hippocampal DG (Smith et al., 1995). Further, chronic unpredictable stress induced a volumetric reduction of hippocampus and MPFC, decreased total dendritic length and spine density, impaired expression of synaptic remodeling proteins (Bessa et al., 2009), undermined hippocampal LTP induction, and attenuated BDNF expression (Qiao et al., 2014). Stress also affects brain expression of other molecules attached to BDNF signaling. In such cases, prenatally stressed rat offspring present decreased CREB and ERK2 mRNA expression in both hippocampus and prefrontal cortex in 3-month olds (Zhu et al., 2016).

Since stress exposure has been described as an environmental factor underpinning neurobiology of mood and anxiety disorders such as depression and PTSD (Post, 1992; Besnard and Sahay, 2016), behavioral and emotional changes characteristic of this disorders could result from stress effects in neuronal plasticity through compromising BDNF expression in specific brain circuits. In accordance with this idea, hippocampal BDNF overexpression succeeded in promoting resilience to stress effects on behavior (Taliaz et al., 2011) and increased dendritic complexity in DG neurons (Tolwani et al., 2002). Further, forebrain BDNF overexpression induced antidepressant and anxiogenic-like effects concomitantly that mimicked stress induced-amygdalar spinogenesis and prevented stress-induced hippocampal dendritic atrophy (Govindarajan et al., 2006). Furthermore, transgenic mice overexpressing the full-length TrkB (trkB.TK+), which had an overactive TrkB-PLCgamma pathway, exhibited reduced anxiety traces, and an antidepressant-like phenotype (Koponen et al., 2004a, 2005), while expression of several plasticity-related genes was found modified (Koponen et al., 2004b). Thereafter, heterozygous BDNF null $\left(\mathrm{BDNF}^{+/}\right.$ ${ }^{-}$) mice, whose BDNF brain levels are decreased approximately by $50 \%$, show increased vulnerability to developing consequences of stress exposure (Lindholm and Castren, 2014).

Therefore, solid deleterious effect of stress upon neural plasticity has been described in several brain regions related to modulation of behavioral consequences of stress. BDNF down regulation in critical brain regions emerging in response to chronic and/or intense ongoing stress exposure is thought to be at least partially responsible for neural plasticity changes. Interestingly, antidepressant treatment, regardless of its primary mechanism of action, is able to reverse stress effects on BDNF levels and brain neurocircuitry related to the regulation of mood and fear (Bessa et al., 2009; Duman et al., 2000; Duman and Monteggia, 2006).

\subsection{BDNF/TrkB signaling participation in antidepressant-induced behavioral effects}

As described previously, independent studies show BDNF and antidepressant treatment acting upon converging signaling pathways. Accordingly, repeated treatment with conventional antidepressants increased BDNF mRNA and changes TrkB levels, particularly in rat hippocampus (Nibuya et al., 1995, 1996; Russo-Neustadt et al., 2000). Human postmortem studies found increased BDNF expression in dentate gyrus, hilus and supragranular regions of antidepressant-treated subjects (Chen et al., 2001a). Both chronic and acute treatment with different classes of antidepressants can activate TrkB (phosphorylation of Y705 and Y816) in structures such as hippocampus, cingulate cortex, and $\mathrm{mPFC}$, respectively in a BDNF-dependent or independent manner (Rantamaki et al., 2007, 2011).

Moreover, a myriad of works consistently exhibit BDNF signalingdependent behavioral and physiological brain changes induced by antidepressants. For instance, desipramine has failed in decreasing immobility time in the forced swimming test in mice, with transient knockdown of BDNF expression in forebrain (Monteggia et al., 2004). The same drug also failed in conditional BDNF knockout mice (Monteggia et al., 2007). Knockdown of BDNF expression, specifically in either DG or CA1 region of hippocampus, prevented antidepressantlike effect of desipramine and citalopram (Adachi et al., 2008). Furthermore, mice overexpressing the nonfunctional dominant-negative isoform of BDNF receptor (TrkB.T1), in the hippocampus and cortical brains structures, were resistant to FLX and IMI effects in the forced swimming test, as were also BDNF haploinsufficient $\left(\mathrm{BDNF}^{+/-}\right)$mice (Saarelainen et al., 2003). On the other hand, direct BDNF infusion into 
the DG and CA3 areas of the hippocampus mimicked the antidepressant-like effect of IMI and FLX treatment in rodents exposed to learned helplessness and completing the forced swimming test (Shirayama et al., 2002).

Besides the behavioral effects, antidepressant-induced plasticity is also affected in impaired TrkB signaling. For example, $\mathrm{BDNF}^{+/-}$mice and those overexpressing TrkB.T1 were resistant to IMI effect in facilitating long-term survival of newborn hippocampal neurons (Sairanen et al., 2005). In addition, mice lacking TrkB in hippocampal neural progenitor cells had impaired neurogenesis, along with resistance to antidepressant treatment on both depression and anxiety paradigms (Li et al., 2008). Accordingly, BDNF/TrkB signaling is supposed to be important for survival and proliferation of hippocampal newborn neurons, and then important for antidepressant efficacy. In fact, Santarelli et al. suggest hippocampal neurogenesis is required for the behavioral effects of antidepressants (Santarelli et al., 2003). Next, Bessa and coworkers found neurogenesis requirement for anxiolytic- but not to antidepressant-like effect of these compounds (Bessa et al., 2009), although studies with nonhuman primates corroborate the neurogenesis-dependent effect of antidepressant action (Perera et al., 2011).

Interestingly, mice carrying a single nucleotide polymorphism in BDNF (V66M) (Chen et al., 2006) show downregulation of this neurotrophin release from the regulated secretory pathway and resistance to antidepressants (Chen et al., 2006; Yu et al., 2012). Accordingly, V66M animals show a multitude of brain alterations, including reduced synaptic plasticity, impaired dendritic complexity, and restricted brain volume in structures such as hippocampus and mPFC (Chen et al., 2006; Pattwell et al., 2012; Yu et al., 2009).

A key pathway for BDNF-induced synaptic plasticity and behavioral changes relies on ERK/CREB signaling (Sweatt, 2001). ERK (extracellular signal-regulated protein kinase) is a member of the mitogenactivated protein kinase (MAPK) family and it has been considered to be a biochemical signal integrator and coincidence detector for coordinating secondary pathways responses to extracellular signals, thus regulating neuronal plasticity (Sweatt, 2001). CREB (cyclic AMP-responsive-element-binding protein) is a transcription factor and downstream target of ERK (Xing et al., 1996). Regarding post-mortem studies, in major depressive subjects decreased CREB levels were found in the temporal cortex, whilst subjects taking antidepressant medications at the time of death had normalized CREB levels (Dowlatshahi et al., 1998). Accordingly, BDNF infusion into DG was able to increase ERK phosphorylation (pERK, which is then activated) in all hippocampal subfields (Shirayama et al., 2002). Furthermore, mBDNF effects were no longer observed in the learned helplessness with previous MAPK inhibitor injected into hippocampus (Shirayama et al., 2002). Similarly, BDNF application to hippocampal slices promoted phosphorylation of MAPK and the inhibition of MAPK prevented BDNF effect on hippocampal synaptic fatigue induced by tetanic stimulation (Gottschalk et al., 1999). Further, peripheral BDNF administration increased its own hippocampal expression, as well as pERK and CREB levels (Schmidt and Duman, 2010).

Similar to BDNF, conventional antidepressants are capable of increasing CREB mRNA and CREB protein levels in hippocampus (Nibuya et al., 1996). Repeated treatment with FLX prevented behavioral consequences of stress and hippocampal changes on pERK and PCREB levels (Qi et al., 2008). Providing a more causal relationship between antidepressant effect and ERK/CREB signaling, Duman and coworkers observed MAPK kinase inhibitor blocked antidepressant-like effect of sertraline and desipramine on forced swimming and tail suspension tests (Duman et al., 2007). In addition, CREB overexpressed in DG was sufficient to produce antidepressant effect on learned helplessness and forced swimming paradigms (Chen et al., 2001b). Moreover, systemic phosphodiesterase inhibitor, which increases cAMP levels, also upregulated hippocampal CREB, BDNF, and TrkB mRNA levels (Nibuya et al., 1996). Using pharmacological inhibition of cAMP catabolism, Nakagawa and colleagues provided evidence for the beneficial effect of
CAMP-CREB signaling on proliferation and survival of hippocampal newborn neurons (Nakagawa et al., 2002a,b). Thus, similar to BDNF, downstream ERK/CREB pathway activation also plays an important role in mediating the therapeutic effect of antidepressants.

In agreement with this idea, activation of cAMP-second messenger signaling increased TrkB and BDNF mRNA levels in cultured astroglial cells (Condorelli et al., 1994). Tao and colleagues, using cultured cortical neurons, observed that $\mathrm{Ca}^{2+}$ triggered-phosphorylation of CREB (CREB binding to $\mathrm{Ca}^{2+}$ response element in the BDNF gene promoter) is responsible for BDNF expression (Tao et al., 1998). Similarly, CREB activation seems to be important to BDNF expression in cultured rat brain astrocytes (Takano et al., 2012). Moreover, treatment with different classes of antidepressant drugs increased pCREB levels and CREmediated gene expression in glial cells, and that effect was blocked with MAPK kinase inhibitor (Takano et al., 2012; Hisaoka et al., 2008). Brain signal integration formed by structure complexes of astroglial cells and pre- and postsynaptic terminals is termed tripartite synapse (Araque et al., 2014; Halassa and Haydon, 2010; Koizumi et al., 2005). Since CREB, CAMP, and BDNF may stimulate each other expression, bidirectional CREB/BDNF interaction in such tripartite organization can play a central role in the positive regulatory loop of BDNF. Thus, sustained BDNF levels are probably maintained at optimal levels to promote neuronal plasticity-dependent therapeutic efficacy of antidepressants (Schmidt and Duman, 2010; Shirayama et al., 2002). Although it is thought that antidepressant treatment requires a couple of weeks to increase brain BDNF levels to then provide therapeutic efficacy, acute antidepressant treatment can transactivate TrkB independently of BDNF or monoamine upregulation and thus also may yield brief bouts of increased BDNF levels to engage ensuing sustained BDNF-TrkB signaling (Rantamaki and Yalcin, 2016). In fact, early state from antidepressant treatment is already able to slightly decrease the psychiatric symptoms, that gradually culminate in the complete and more obvious long-term efficacy (Rantamaki and Yalcin, 2016). Thus, it is noteworthy to highlight that transitory increment of BDNF levels or TrkB activation may be important to initially change network function and to further trigger network rewiring-based long-term effects of antidepressants (Rantamaki and Yalcin, 2016).

\section{TrkB/p75 opposite mechanism of action}

Similar to BDNF, proBDNF and BDNF-propeptide can be stored in dense core vesicles from presynaptic terminals and secreted from a regulated pathway in an activity-dependent fashion (Mizui et al., 2015; Lu, 2003b; Guo et al., 2016; Dieni et al., 2012). Both mBDNF and proBDNF bind to different receptors and elicit different physiological responses. The released proBDNF activates exclusively p75/sortilin coreceptor complex (p75R), although BDNF, as well as other neurotrophins, is capable of binding with lower affinity to p75R (Meeker and Williams, 2015). The activation of $\mathrm{p} 75 \mathrm{R}$ promotes programmed death in a wide variety of neuronal populations, including sympathetic (Bamji et al., 1998; Linggi et al., 2005; Teng et al., 2005), motor (Sedel et al., 1999), retinal (Frade et al., 1996), and hippocampal neurons (Volosin et al., 2008). Furthermore, activation of p75R by proBDNF promotes hippocampal LTD (long-term depression) (Martinowich et al., 2012; Woo et al., 2005). Based on a Hebbian plasticity model, LTP specific input would favor synaptic strength, whereas LTD would impair it, thereby avoiding an uncontrollable runaway excitation (Pozo and Goda, 2010). Mice with one BDNF mutant allele, in which proconvertase/furin cleavage site was mutated, presented an increase of proBDNF hippocampal levels, reduced dendritic arborization and spine density, decreased hippocampal volume, impairment in basal synaptic transmission, and enhanced LTD (Yang et al., 2014). In aged mice, intra-hippocampal treatment with proBDNF decreased the number of hippocampal newborn cells, whilst infusion of anti-proBDNF antibody counteracted such effects (Chen et al., 2016). Conversely, in an opposite way to $\mathrm{mBDNF}$, proBDNF is suggested to inhibit prolonged firing 


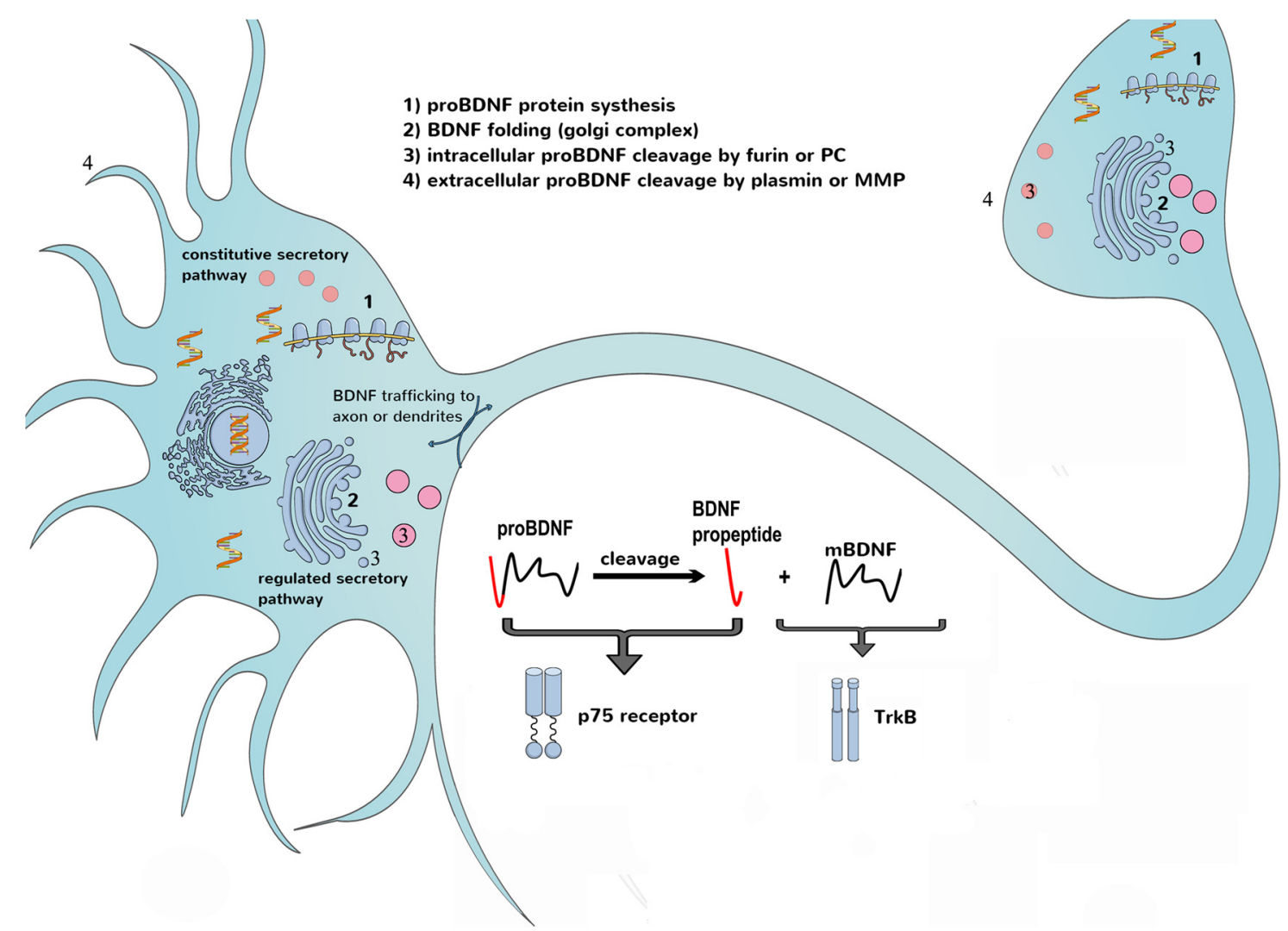

Fig. 1. mBDNF/proBDNF opposite mechanism of action. BDNF transcripts are translated in the endoplasmic reticulum as a precursor protein called proBDNF, which may be further cleaved within the Golgi complex by furin or within vesicles by pro-protein convertases. proBDNF can also be secreted into synaptic cleft, where it may be cleaved by metalloproteinases and/or plasmin, giving rise to the mature BDNF (mBDNF) and the protein portion related to pro-domain (BDNF propeptide). BDNF is usually sorted into large dense core vesicles to be released in an activity-dependent fashion from the regulated pathway, but unsuitable folding of the mature domain sorts BDNF to be released from the constitutive pathway. As observed with BDNF, BDNF pro-peptide, as well as proBDNF, can also be secreted from the regulated pathway. mBDNF binds to TrkB, whereas proBDNF and BDNF propeptide may bind to the p75/sortilin co-receptor complex.

activity through $\mathrm{p} 75 \mathrm{R}$, negatively regulating the release of glutamate (Gibon et al., 2016). Therefore, p75 activation by proBDNF would convey opposing mechanisms to TrkB activation by mBDNF (see Fig. 1).

Interestingly, the protein portion corresponding only to the prodomain is also suggested to trigger the p75R signaling, since BDNFpropeptide enhanced LTD in a p75R-dependent manner (Mizui et al., 2015). BDNF-propeptide obtained from the polymorphic gene sequence V66M has been able to induce growth cone retraction also in a $\mathrm{p} 75 \mathrm{R}$ dependent manner (Anastasia et al., 2013). Indeed, exposition of cultured hippocampal neurons to the BDNF-propeptide reduced dendritic spine density, an effect that required caspase- 3 and release of cytosolic cytochrome c (Guo et al., 2016).

The activation of p75R triggers a signaling cascade involving JNK (jun $N$-terminal kinase) activation and apoptosis (Kraemer et al., 2014). JNK activation in response to p75R activation was observed in sympathetic (Bamji et al., 1998) and hippocampal neurons (Friedman, 2000), whereas JNK inhibition prevented apoptosis induction from p75R activation in many cell types (Friedman, 2000; Harrington et al., 2002; Kenchappa et al., 2010; Yeiser et al., 2004; Yoon et al., 1998). Overexpression of $\mathrm{p} 75 \mathrm{R}$ in cortical neurons was also able to promote JNK activation (Bhakar et al., 2003). In addition, JNK signaling, associated with $\mathrm{p} 75 \mathrm{R}$ activation, involves mainly phosphorylation of p53 tumor suppressor protein (Fuchs et al., 1998; Oleinik et al., 2007). In line with these data, p75R activation and death of sympathetic neurons are associated with increased expression of p53 (Linggi et al., 2005; Aloyz et al., 1998), with phosphorylation of pro-apoptotic proteins such as Bad and Bim (Bhakar et al., 2003; Becker et al., 2004), with mitochondrial cytochrome c release (Bhakar et al., 2003) and with cleavage of pro-caspase 3,6,7, and 9 to active caspase protein (Bhakar et al.,

\section{3; Tabassum et al., 2003).}

\section{The continuum-sorting hypothesis}

The present hypothesis postulates that antidepressant treatment might be able to increase, beyond mBDNF expression, proBDNF or at least the propeptide levels, since mBDNF production is tied to previous proBDNF making. Scarce evidence goes toward this proposal, inasmuch as very few studies address the functional role of proBDNF or propeptide as a regulator of behavioral consequences of stress (see Table 1). Since chronic antidepressant treatment with IMI and FLX increased not only neurogenesis, but also apoptosis of DG neurons (Sairanen et al., 2005), it is plausible to consider that such opposing mechanisms in cellular plasticity are taking place to convey the behavioral effects as an end product succeeding the neuronal turnover. Although this work does not address the mechanism behind apoptosis induced by antidepressant treatment, it is plausible to conject the involvement of proBDNF/propeptide/p75R signaling. Indeed, antidepressant treatment with escitalopram or duloxetine reversed the lower serum levels of mBDNF and tPA found in depressed patients, just as reversed the previous increased levels of proBDNF (Jiang et al., 2017). However, Yoshimura et al. (2014) found depressed patients with lower serum mBDNF and unchanged proBDNF levels compared to healthy controls, whereas both proBDNF and mBDNF serum levels had no discrepancy between drug (fluvoxamine) responder and nonresponder (Yoshimura et al., 2014). Curiously, in this study, fluvoxamine was unable to change either mBDNF or proBDNF serum levels and fluvoxamine plasma levels were not even correlated with depressive scores and mBDNF levels (Yoshimura et al., 2014). In addition, repeated treatment with 
Table 1

Procedures related to MDD and PTSD influencing proBDNF signaling. Every work we have knowledge of using tools related to proBDNF signaling was briefly described, either concerning animal models or clinical research related to depression or PTSD. Notes: MDD - major depressive disorder; tPA - tissue plasminogen activator; JNK - c-Jun N-terminal kinases; mPFC - medial prefrontal cortex; Nac - nucleus accumbens.

\begin{tabular}{|c|c|c|c|}
\hline \multicolumn{4}{|c|}{ POSTTRAUMATIC STRESS DISORDER } \\
\hline protocol & Area & Effect & Reference \\
\hline \multirow[t]{2}{*}{ contextual fear conditioning } & CA1 & $\begin{array}{l}\text { proBDNF levels was increased } 6 \mathrm{~h} \text { after extinction and decreased } 6 \mathrm{~h} \text { after conditioning } \\
\text { protocol }\end{array}$ & $\begin{array}{l}\text { Barnes and Thomas } \\
(2008)\end{array}$ \\
\hline & dorsal hippocampus & $\begin{array}{l}\text { infusion of an inhibitor of tissue plasminogen activator before extinction increased } \\
\text { proBDNF levels } 6 \mathrm{~h} \text { after and decreased conditioned response during recall test } 1 \text { day after } \\
\text { infusion of a tPA inhibitor before conditioning decreased further freezing behavior }\end{array}$ & \\
\hline inhibitory avoidance task & CA1 & infusion of JNK inhibitor impaired extinction of inhibitory avoidance memory & Bevilaqua et al. (2007) \\
\hline \multicolumn{4}{|l|}{ DEPRESSION } \\
\hline \multirow[t]{6}{*}{ clinical research } & serum and lymphocites & proBDNF, p75 and sortilin levels was found increased in MDD patients & Zhou et al. (2013) \\
\hline & serum & $\begin{array}{l}\text { escitalopram and duloxetine reversed the lower levels of BDNF and tPA and the higher } \\
\text { levels of proBDNF found in depressed patients }\end{array}$ & Jiang et al. (2017) \\
\hline & & lower BDNF and unchanged proBDNF levels were found in depressed patients. & Yoshimura et al. \\
\hline & & Fluvoxamine was not able to change either BDNF or proBDNF levels and even its plasma & $(2014)$ \\
\hline & & $\begin{array}{l}\text { levels have not been correlated with depressive scores. BDNF and proBDNF levels were not } \\
\text { found different between drug responder and no responders. }\end{array}$ & \\
\hline & blood samples & premenopausal women with MDD had increased plasminogen activator inhibitor- 1 levels & $\begin{array}{l}\text { Eskandari et al., } \\
\text { (2005) }\end{array}$ \\
\hline \multirow[t]{2}{*}{ postmortem } & parietal cortex & lower BDNF and higher BDNF-propeptide levels were found in depressed patients & Yang et al. (2017a) \\
\hline & cerebellum & $\begin{array}{l}\text { levels of proBDNF and BDNF-propeptides had decreased while BDNF levels was unchanged } \\
\text { in depressed patients }\end{array}$ & \\
\hline \multirow[t]{4}{*}{ learned helplessness } & $\mathrm{Nac}$ & helpless animals had decreased proBDNF levels & $\begin{array}{l}\text { Shirayama et al. } \\
\text { (2015) }\end{array}$ \\
\hline & $\mathrm{mPFC}$ & helpless animals had increased proBDNF levels & \\
\hline & & susceptible animals had increased proBDNF and BDNF pro-peptide levels & Yang et al. (2016) \\
\hline & $\mathrm{Nac}$ & susceptible animals had decreased proBDNF and BDNF pro-peptide levels & \\
\hline \multirow[t]{7}{*}{$\begin{array}{l}\text { unpredictable chronic mild } \\
\text { stress }\end{array}$} & i.c.v or i.p & $\begin{array}{l}\text { anti-proBDNF antibody infusion abrogated stress effect on sucrose or forced swimming } \\
\text { test, beyond preventing reductions in dendritic spine lenght }\end{array}$ & Bai et al. (2016) \\
\hline & $\begin{array}{l}\text { neocortex and } \\
\text { hippocampus }\end{array}$ & stress exposure increased levels of proBDNF, p75 and sortilin & \\
\hline & & stress exposure increased sortilin levels & Ruan et al. (2016) \\
\hline & PFC & $\begin{array}{l}\text { stress exposure increased proBDNF and plasminogen activator inhibitor-1 levels and } \\
\text { sertraline chronic treatment increased tPA levels }\end{array}$ & Tang et al. (2015) \\
\hline & hippocampus & stress exposure increased proBDNF and decreased tPA levels & Zhang et al. (2016) \\
\hline & anterior cingulate cortex & $\begin{array}{l}\text { infusion of anti-proBDNF antibody refrained pro-depressive-like symptoms and the } \\
\text { decrease of BDNF levels in mice }\end{array}$ & (Yang et al. 2017b) \\
\hline & hippocampal CA1 & $\begin{array}{l}\text { infusion of proBDNF mimicked stress effect, although stress have not increased proBDNF } \\
\text { levels }\end{array}$ & Qiao et al. (2017) \\
\hline \multirow{5}{*}{$\begin{array}{l}\text { tail suspension test } \\
\text { voluntary physical activity } \\
\text { enriched environment } \\
\text { electroconvulsive shocks }\end{array}$} & p75 knockout & knockout animal showed antidepressant-like profile & Catts et al. (2008) \\
\hline & hippocampus & increased p11 and tPA mRNA expression & Sartori et al. (2011) \\
\hline & & up-regulated matrix metalloproteinase- 9 & Cao et al. (2014) \\
\hline & & increased furin, prohormone convertase 1 and tPA mRNA expression & Segawa et al. (2013) \\
\hline & $\begin{array}{l}\text { dorsal/ventral } \\
\text { hippocampus }\end{array}$ & increased TrkB and BDNF levels, but proBDNF have been unchanged & Enomoto et al. (2017) \\
\hline
\end{tabular}

sertraline was able to increase tPA brain levels in animals (Tang et al., 2015). Although clinical reports address a miscellaneous effect of antidepressants on proBDNF level changes, evidence generally advocates that antidepressants seemingly may modulate both signaling attached to $\mathrm{mBDNF}$ and proBDNF. Hereupon, more studies are necessary to request whether proBDNF and/or BDNF-propeptide levels are in fact modulated by antidepressants and it would have a functional role on neuronal turnover and remodeling. For example, BDNF-propeptide levels were never verified regarding whether its levels were changed by antidepressant and additional studies concerning specific brain areas is also required. Therefore, previous conjecture remains to be investigated.

In the present review, we propose that therapeutic efficacy of antidepressants would arise from a facilitation of synaptic remodeling and neuronal turnover, with both $\mathrm{mBDNF}$ and proBDNF/propeptide playing important roles in this scenario (see Fig. 2). mBDNF/TrkB and proBDNF/propeptide/p75R, respectively through LTP and LTD plasticity, would sort probability of neuronal survival from synaptic stabilization, taking into account overall activity of neuron from each particular synaptic strength. Usually only mBDNF's role in competitive selection of neurons has been considered as a factor for the survival of synaptic contacts (Castren, 2005), while proBDNF-induced pruning was never supposed as a cooperator for that antidepressant-induced competition.

The literature has focused on the functional role of LTP in learning and memory. However, LTD also plays a major role in these cognitive processes. For example, mice lacking GluN2B have impaired hippocampal LTD, decreased dendritic spine density, cognitive deficits in the Morris water maze, decline in the spontaneous alternation on trial Tmaze task and lower levels of freezing behavior after trace fear conditioning (Brigman et al., 2010). Accordingly, LTD and spatial memory consolidation were concomitantly damaged with pharmacological tools, such as GluN2B antagonist or AMPA receptor endocytosis blocker, infused systemically, or into the dorsal hippocampus (Ge et al., 2010). That same research group verified that both drugs decrease the performance on a spatial reversal learning memory task, while stress facilitated it as well as enhanced hippocampal LTD (Dong et al., 2013). Besides, animals trained with platform on Morris water maze, but not untrained animals, had enhanced hippocampal LTD in vivo (Dong et al., 2013). Similar results was obtained with animals trained in exploratory 


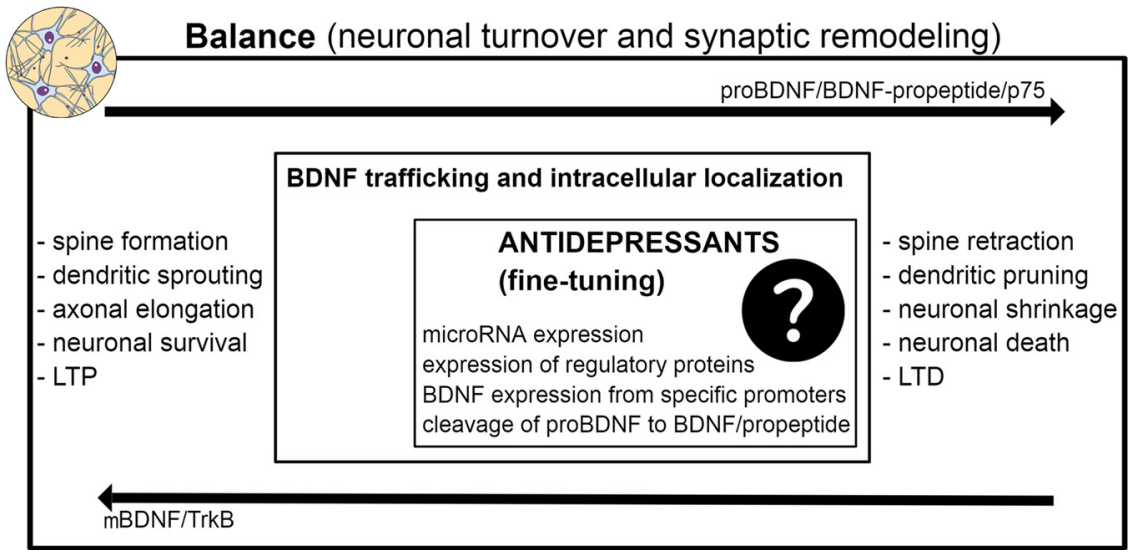

Fig. 2. Continuum-sorting hypothesis in antidepressant drug's perspective - part 1. Although TrkB/p75 signaling display a conflicting functional role upon neuronal plasticity, both pathways might have a synergistic effect to promote activity-dependent neuronal turnover and remodeling. This antidepressant-dependent, fine-tuning mechanism would be biologically relevant regarding homeostatic balance between the network efficacy and energy expenditure that underpin the best coping strategies to environmental contingencies. behavior around a novel environment (Manahan-Vaughan and Braunewell, 1999).

Maturation of several brain regions is subordinated to activitydriven synaptic refinements, such as elimination of axonal connections and spine pruning (input disconnection), while redundant neuro-circuitry is found in immature CNS (Lichtman and Colman, 2000). Therefore, adult brain is less labile while spines become stable in adulthood (Grutzendler et al., 2002). Selective pressure likely favors adult rigidity of neuronal plasticity, at the expense of cognitive flexibility, to protect circuit engram translating older memories from more recent memory, thus avoiding the risk of overwriting. With the advent of complex social networks and robust psychological challenges, impairment of cognitive flexibility became a huge burden to psychological human health. Cognitive flexibility would be important to drive behavior in accordance with a myriad of environmental contingences, in order to make a better outcome of decision-making (Anacker and Hen, 2017).

Acute stress may facilitate reversal learning and behavioral flexibility, thus reducing interference of previously acquired memories over newer ones, and/or by reducing the interference of emotionally-neutral memories (Dong et al., 2013). In fact, traumatic and intense acute stress enable cognitive flexibility, thereby enhancing fear memory and originating post-traumatic stress disorder (PTSD) symptoms (VanElzakker et al., 2014). However, cognitive and behavioral flexibility highlighted by limbic structures are also crucial to facilitate extinction of conditioned fear memory (Maren et al., 2013). On the other hand, absence of cognitive flexibility would impair resilience to neuroplastic changes induced by enduring stress exposure, finally culminating in disorders such as depression (Southwick et al., 2005). Antidepressant treatment is likely to restore CNS neuroplasticity and cognitive flexibility to developmental levels (Castren and Antila, 2017). As a consequence, we suggest that this process of restauration is paved by mBDNF and proBDNF/propeptide in a stepwise effect upon neuronal turnover and spine remodeling. Consequently, overwhelming environmental contingencies become manageable, by allowing unpleasant and negative memories to be efficiently replaced by neutral or pleasant emotional salience.

The complexity behind BDNF trafficking and sorting via a range of complex molecular arrangements and specific environmental requirements could be a key tool to cognitive flexibility. In fact, the biological functional role of different mRNAs encoding same protein is still not fully understood, but evidence points to a modulatory influence in BDNF trafficking and specific environmental contingences acting on specific BDNF transcripts. For instance, it was described that short 3'UTR mRNAs restrict BDNF transcripts to the soma while long 3'UTR mRNAs are restricted to dendrites, where local translation occurs (An et al., 2008). Mutant mice with a truncated long 3'UTR exhibited deficits in dendritic spine enlargement and pruning (An et al., 2008), whereas cultured hippocampal neurons supporting long 3'UTR mRNA overexpression showed an enhancement of spine pruning and maturation (Orefice et al., 2013). Accordingly, long BDNF 3'UTR is implicated in suppressing translation whilst short 3'UTR undergoes active translation to maintain BDNF basal levels; and upon neuronal activity the long 3'UTR is associated to quick translation (Lau et al., 2010). However, deletion of cytoplasmic polyadenylation element-like sequences in the short 3'UTR negatively impacts dendritic targeting of BDNF mRNA, either in a constitutive or activity-dependent way (Oe and Yoneda, 2010). In addition, under depolarizing conditions, either obtained with $\mathrm{KCl}$ exposition on hippocampal neuronal culture or with seizure induced in vivo, it was verified that both short and long 3'UTRs could be taken by distal dendrites (Vicario et al., 2015). Long and short 3'UTRs appear to use distinct sets of RNA-binding proteins to dendrite targeting from different stimuli (Vicario et al., 2015). BDNF transcript exons 1 and 4 are found confined to soma and proximal dendrites, while exon 2 and 6 lengthen into distal dendrites in response to activity (Pattabiraman et al., 2005; Chiaruttini et al., 2008) to adjust the morphology of local dendrites (Baj et al., 2011). In fact, Steward and Schuman (2001) provided evidence that dendritic protein synthesis linked to mRNA trafficking is important to modulate synaptic strength and plasticity (Steward and Schuman, 2001). Therefore, with a more recent glimpse of overview, the somatic and/or dendritic targeting is probably more confined to the general diversity of BDNF transcripts irrespective of polyadenylation signal, whilst both short and long 3'UTRs can be targeted to the dendrites on demand, in response to synaptic activity.

In 2008, Tongiorgi et al hypothesized that segregation of BDNF transcripts yields a "spatial code", the modulation of which is engendered as a mechanism to provide BDNF availability to different subcellular compartments during neuronal plasticity (Tongiorgi, 2008). Considering proBDNF, short $3^{\prime}$ UTR BDNF mRNA translated in soma was mainly found released in mBDNF form independent of action potentials inasmuch as long 3'UTR BDNF mRNA translated in dendrites was mostly released as proBDNF upon neuronal activity (Orefice et al., 2016). The release of proBDNF from dendrites increased spine pruning as well as spine head enlargement (maturation) in cultured hippocampal neurons, partially by p75R; or proBDNF could be extracellularly cleaved to mBDNF to induce spine maturation (Orefice et al., 2016). Therefore, we suggest that "spatial code" yielded by BDNF transcripts would account for both mBDNF and proBDNF/propeptide as key regulators of synaptic remodeling and synaptic competition, thus favoring cognitive flexibility. Accordingly, mBDNF and proBDNF have already been described as "reward" and "punishment" signals for inactive and active synaptic terminals at developing neuromuscular synapses ( $\mathrm{Je}$ et al., 2012, 2013). Thereafter, refinement of neuronal connectivity from synaptic transmission desynchronization in neighbor synapses is also modulated by proBDNF/p75 signalling (Winnubst et al., 2015).

Different kinds of stimuli can modulate and promote BDNF mRNA compartmentalization. For example, mice stimulated with repeated 
physical exercise had global BDNF mRNA levels increased in CA3 neuronal somata and dendrites (Baj et al., 2012). Repeated treatment with fluoxetine or reboxetine mimicks physical exercise and increases BDNF mRNA levels in CA1 and DG somata (Baj et al., 2012). Specifically BDNF mRNA transcript 6 , in neuronal somata and dendrites of whole hippocampus, was prominently increased following physical exercise, whereas pharmacological treatment specifically increased this transcript in neuronal somata and dendrites in the CA3 area; and alterations in BDNF mRNA transcript 4 were quite subtle (Baj et al., 2012). Transcripts containing exon 6 broadly accounted for the higher level in dendritic labeling for BDNF in depolarized cultured hippocampal neurons (Baj et al., 2012). In fact, in the hippocampus and cortex, BDNF mRNA transcripts are trafficked to distal dendrites in response to several stimuli (Capsoni et al., 1999; Righi et al., 2000; Tongiorgi et al., 2004, 1997). In addition, KCl-induced membrane depolarization regulated exon-specific BDNF transcripts in cultured cortical neurons (Tao et al., 1998), as did kainate-induced seizures in the adult rat hippocampus (Aid et al., 2007; Timmusk et al., 1993; Metsis et al., 1993) and cerebral cortex (Metsis et al., 1993); and pilocarpineinduced seizures in parietal cortex (Metsis et al., 1993). Stress-dependent, exon-specific BDNF transcript changes were also noticed in different animal models (Marmigere et al., 2003; Nair et al., 2007). Furthermore, 10 Gy-irradiated mice demonstrated impaired learning and memory, and decreased exon-specific BDNF transcript expression (Son et al., 2015). Besides, microRNAs can modulate BDNF expression and the presence of specific BDNF 3'UTR gene variants also can control microRNAs-BDNF binding (Caputo et al., 2011). In addition, modulatory complexity around expression of microRNAs is also evidenced, since microRNA expression can also be cell-specific, temporarily controlled (Smirnova et al., 2005), and induced in specific brain areas by stressors such as restraint stress (Rinaldi et al., 2010).

Different patterns of BDNF transcript expression have been found from different brain regions during embryonic or postnatal development (Aid et al., 2007; Timmusk et al., 1993, 1994). In addition, distinct patterns of human BDNF transcript expression have also been found in several brain regions and, in contrast to rodents, natural noncoding antisense RNAs are transcribed from the antiBDNF gene locus (Pruunsild et al., 2007). Surprisingly, it was revealed that BDNF and antiBDNF transcripts give rise to double-stranded RNA complexes, suggesting even a greater fine-tuning regulation of BDNF expression in humans (Pruunsild et al., 2007). With this prospect, complexity around BDNF production, gene transcript, and post transcript regulation is likely intrinsically related to the evolutionary value of its crucial influence on neural development and adult neuroplasticity. Thus, disruption in transcript regulation of the BDNF gene would provide an impairment in the ability to face cognitive challenges and psychological stressors, which in turn would lead to maladaptive behavioral responses to stress, such as depression and PTSD.

As discussed above, multiple mechanisms may be involved in BDNF expression, trafficking, and intracellular localization, e.g. teeming promoters, microRNA control, regulated cleavage of proBDNF to mBDNF, whose operational demands lies under specific tissue requirements and environmental contingencies (drug and stress exposure). In addition, several proteins are described to influence BDNF trafficking, examples include, but are not limited to: $\alpha$-synuclein (Fang et al., 2017), huntingtin (Ma et al., 2010), translin (Wu et al., 2011), and the heterogeneous nuclear ribonucleoprotein CArG Box binding Factor A (Raju et al., 2011). A recent study has depicted that mBDNF capacity of compromising hippocampal LTD is impaired inasmuch as BDNF-propeptide is able to bind to mBDNF with a higher affinity (Uegaki et al., 2017). Complementary, TrkB gene can be translated into four versions, the full-length $\mathrm{TrkB}$ and the truncated versions originated from splice variants (Sasi et al., 2017). mBDNF binds to truncated variants as well, but does not activate downstream kinase cascades, thus presenting an additional point to modulate mBDNF/TrkB signaling (Sasi et al., 2017). Therefore, despite the fact that mBDNF and
proBDNF/propeptide usually induce dichotomous neuroplastic effects individually (for review see (Gibon and Barker, 2017)), an orchestrated interaction promoting and selecting specific connections is not an implausible scenario.

In conclusion, complexity around BDNF gene expression, posttranscriptional regulation, cell trafficking, and the post-translational targets with bidirectional consequences would provide for a myriad of possibilities with respect to promoting neuroplasticity. In addition, as observed with NGF in some particular conditions (specially without sortilin coupling to P75R) (Nykjaer et al., 2005), it is possible that both TrkB and p75R might also act together in a synergic way to favor neuroplasticity; and BDNF even might act on P75R to induce neuronal death in case of TrkB absence (Friedman, 2000). Further highlighting the complexity behind both p75R and TrkB signaling, p75R signaling may also promote cell survival whereas TrkB can lead to cell death (for review see (Kalb, 2005)). Accordingly, the landscape of continuum concerning the neuroplastic opportunities in response to the TrkB and p75R balance is enlarged further, expanding the beyond good and evil perspective. Thus, greater cognitive flexibility is a by-product likely of neuroplasticity, which drives a larger behavioral repertoire to face different environmental and psychological challenges. Then like a computer, which interprets and performs an infinity of tasks in binary language, the continuum-sorting hypothesis proposes mBDNF/ proBDNF and/or mBDNF/propeptide as neuroplasticity classifier agents. They are arranged with opposite (not always) functional roles, that under different environmental demands and neuronal microenvironments make feasible a continuum of possibilities concerning remodeling and neuronal turnover. In fact, extracellular mBDNF/ proBDNF ratio is controlled by neuronal activity, since high-frequency stimulation, but not low frequency, co-released tPA (tissue plasminogen activator, that catalyzes plasminogen conversion to plasmin) along with proBDNF (Nagappan et al., 2009). Since antidepressants can modulate neuronal activity, they may also subjugate the balance of mBDNF/ proBDNF and/or mBDNF/propeptide to promote more efficient neuronal networking, thus favoring neuroplasticity on demand. It is worth noting that detrimental stimuli, such as status epilepticus, can also change the balance of hippocampal TrkB/p75R signaling by switching TrkB towards p75R expression and then favoring either mBDNF or proBDNF receptor binding (Unsain et al., 2008). In this scenario, the antidepressants become a type of "organizer", by allowing the (re) balance between TrkB and p75R signaling, by amplifying neuroplastic possibilities toward fine-tuning of neuronal networking on demand of contingencies and specific requirements. The myriad of probability concerning neuronal turnover and remodeling is mandatory to the "continuum-sorting hypothesis" and is also attached to the assumption that many checkpoints that balance the mBDNF/proBDNF and/or $\mathrm{BDNF} /$ propeptide ratio might be tampered with via antidepressant treatment (see Fig. 2).

An interplay, or synergistic effect, between pharmacological and cognitive therapies is usually supported (Castren, 2013) and could be interpreted considering aspects of therapeutic antidepressant effect relying on a continuum-sorting. Cognitive therapy, by gradually modifying interpretative emotional aspects of psychological challenges, would allow a better targeting of neuronal connections (involved with regulation of behavioral response to stress) that are worth maintaining. Continuum-sorting hypothesis encouraging additional data could also help to explain why antidepressants improve apparently different conditions, since benefit from mBDNF/proBDNF and or mBDNF/propeptide boosting neuronal remodeling or neuronal turnover probably occur widely in the CNS.

\subsection{Posttraumatic stress disorder}

PTSD is a maladaptive behavioral response to traumatic exposure as consequence of an impairment in the extinction of conditioned fear memory and overgeneralization of ambiguous clues erroneously 
foretelling an unpleasant consequence (Besnard and Sahay, 2016). Notably, pattern separation, a neuronal processing whose similar pattern of inputs are converted into distinct representations, has been established to discriminate highly similar contextual and temporal clues (Aimone et al., 2006). Therefore, an impairment of pattern separation in favor of pattern completion, a neuronal processing by which complete pattern of activity is recovered on partial representations, is regarded part of the neurobiology of PTSD. Pattern separation hypothesis states that young adult-newborn dentate granule neurons of hippocampus put forward feedback inhibition to order sparseness of DG mossy fiber activity on CA3 neurons, thus disambiguating memories that are contextually and temporally similar (McAvoy et al., 2015).

BDNF is supposed to be important to modulate pattern separation, since its performance in the DG was required for consolidation of resembling spatial representations, whereas BDNF infused directly into DG was enough to improve spatial discrimination of similar representations (Bekinschtein et al., 2013). Rodent BDNF levels were also increased in the DG after similar spatial representations exposure (Bekinschtein et al., 2013). Moreover, lentiviral approach reducing DG neurogenesis levels of adult male rats prevented BDNF effect of strengthening discrimination of similar spatial representations (Bekinschtein et al., 2014). Nevertheless, the role of proBDNF signaling in pattern separation has never been studied.

Although no work has surrounded the relevance of neuronal turnover on pattern separation, we propose that including DG neuronal death in parallel with neurogenesis might promote a better computational modeling to explain memory interference of ambiguous contingencies. Hebbian learning might rate neuronal survival using proBDNF/mBDNF tools to achieve optimum homeostatic balance between pattern completion and separation to optimize the coping behavior or avoid any hazard attack. Therefore, the final product would be an efficient appraisal and discernment of expectancy with which a cue predicts a harmful outcome. In case of emotional positive valence with evolutionary adaptive relevance, the same rules are valid.

A more comprehensive hypothesis based on memory resolution and autonomous coding units of dentate granule neurons proposes newborn neurons affecting multiple aspects of memory formation (Aimone et al., 2011). Coherent with the memory resolution mechanism, both immature and mature hippocampal granule neurons provide a memory engram with older neurons encoding familiar memories and younger neurons encoding novel features (Aimone and Gage, 2011). In fact, studies using pharmacological or molecular tools to modulate neurogenesis in hippocampus show facilitation or deficit in extinction memory, respectively concerning increase and decrease of newborn cells levels (Hung et al., 2015; Ren et al., 2013; Pan et al., 2012; Deng et al., 2009). Evidence also suggest that quality of extinguishing memory depends on proBDNF/JNK signaling, which, as previously described, is a pathway important for neuronal apoptosis and synaptic bluntness. In this regard, Barnes and coworkers found that, six hours after extinction protocol, animals showed a $250 \%$ increase in proBDNF levels in the hippocampal CA1 area (Barnes and Thomas, 2008). In addition, tPA blocker infused into hippocampus potentiated memory extinction on contextual fear conditioning (Barnes and Thomas, 2008). In another work, Bevilaqua and coworkers found that the JNK inhibitor infused into hippocampus was able to impair the extinction memory on the passive avoidance model (Bevilaqua et al., 2007). In accordance with these data, the hippocampus is the structure where proBDNF, BDNF-propeptide, p75R and JNK expression is more prominent (Yang et al., 2009; Dieni et al., 2012; Coffey, 2014).

PTSD patients might probably have both negative and positive outcomes concerning neuronal plasticity in fear-circuitry, since a deficit of extinction memory and overgeneralization or exacerbated fear memory (intrusive memories) are found. In this case, neuronal turnover would be necessary to resolve symptoms, since a broad and complex reorganization of neuronal fear-circuitry would be required. In this context, $\mathrm{mBDNF} / \mathrm{TrkB}$ signaling could play a role in restoring impaired extinction memory, whereas proBDNF/p75 signaling would be important to disconnect neuro-circuitry of fear-conditioned memories. Altogether, neuronal turnover would be important to restore neuronal homeostasis of fear circuitry in accordance with environmental challenges and contingences. Accordingly, a confluence of data regarding $\mathrm{mBDNF} /$ proBDNF signaling involvement with some perspective of fear memory suggests a midway in which antidepressants might work. Indeed antidepressants, mainly SSRI, are the first line of pharmacological approach to treat PTSD symptoms (Warner et al., 2013). Basic research also suggests that concurrent antidepressant treatment and extinction protocol preclude the recovery of conditioned fear responses (Karpova et al., 2011; Popova et al., 2014). Therefore, as part of the search for most effective treatments for PTSD symptoms, it is essential to study the relevance of turnover upon neuronal circuitry associated to conditioned fear memory.

\subsection{Depression}

Depression symptoms are closely tied to stress exposure. Initial episodes of stress give rise to subtle mood changes, which in consequence of stress recurrence could emerge spontaneously and with more disabling changes (Post, 1992), and a negative correlation between BDNF signaling and depression symptoms have been described, for review see (Castren and Kojima, 2017; Castren and Rantamaki, 2010). However, studies approaching the relationship between proBDNF and depression are beginning to arise. Regarding depression, the dichotomy between the mBDNF/TrkB and proBDNF/p75 functional roles seems to be decisive. Depressed patients show increased proBDNF, p75, and sortilin protein levels in both serum and lymphocyte samples (Zhou et al., 2013). Accordingly, patients with depression had lower serum levels of tPA and mBDNF, whilst TrkB, proBDNF, and p75 were increased (Jiang et al., 2017). Recently, in a post mortem study, lower mBDNF levels were found in the parietal cortex of depressed patients, and conversely a higher level of BDNF-propeptide; although in cerebellum the levels of proBDNF/propeptide was found decreased and mBDNF was unaltered (Yang et al., 2017a). Mildly depressed women in clinical remission had higher serum levels of PAI-1, which is the major endogenous tPA inhibitor (Eskandari et al., 2005).

Rats submitted to the learned helplessness model showed increased protein levels of proBDNF and propeptide in $\mathrm{MPFC}$, increased propeptide levels in the hippocampal CA3 area, and decreased levels of both proBDNF and propeptide in nucleus accumbens (Shirayama et al., 2015; Yang et al., 2016). Chronic unpredictable stress (CUS) decreased murine sucrose intake (considered a depressive-like behavior) as well as increased proBDNF levels of neocortex and hippocampus, while FLX treatment prevented all the mentioned changes (Bai et al., 2016). CUS also increased proBDNF, p75R, sortilin protein, and mRNA levels in both neocortex and hippocampus, whereas systemic or intracerebroventricular infusion of anti-proBDNF antibody prevented the behavioral consequences of stress (Bai et al., 2016). Intracerebroventricular infusion of adenovirus vector carrying proBDNF sequence increased immobility behavior on FST (Bai et al., 2016). In addition, anti-proBDNF antibody infused into anterior cingulate cortex impeded pro-anxiogenic, pro-depressive and the decrease of cortical and hippocampal BDNF mRNA levels induced by CUS, but anti-BDNF antibody was not able to enhance stress effects (Yang et al., 2017b). Infusion of BDNF into the CA1 area of hippocampus precluded CUSinduced depressive-like behavior, preventing the stress effect of decreasing CA1 hippocampal spine density and the decrease in hippocampal levels of postsynaptic proteins of the excitatory synapse, such as postsynaptic density protein 95 , kalirin-7, and NMDA receptor subunit NR2B (Qiao et al., 2017). In addition, infusion of proBDNF into hippocampal CA1 mimicked all these previous effects induced by CUS (Qiao et al., 2017). However in this case, CUS caused decreased hippocampal levels of BDNF, but proBDNF were not increased (Qiao et al., 2017). 


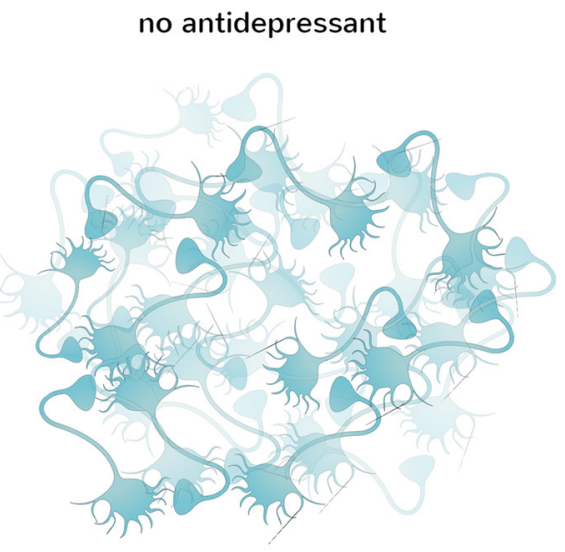

neuronal and synaptic inflexibility

- cognitive rigidity

- worse decision-making

- pathological consequences from

psychological stress

enviromental challenges
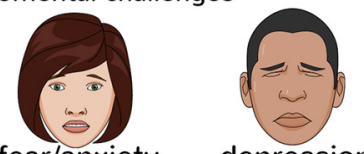
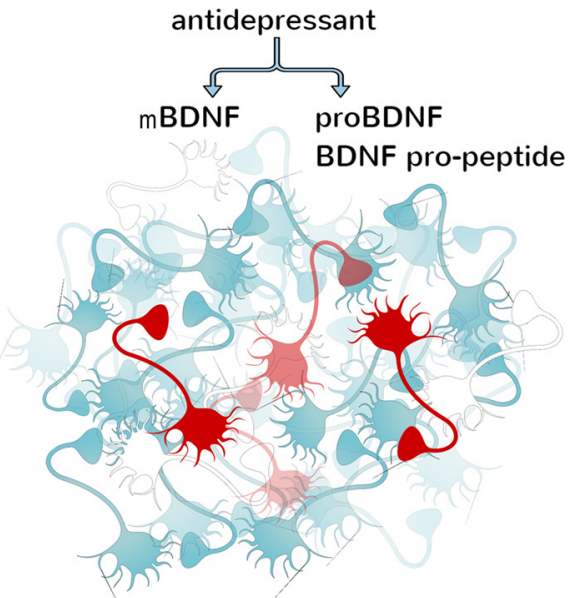

neuronal turnover/synaptic remodeling

- cognitive flexibility

- better decision-making

- resilience to

psychological stress

enviromental challenges

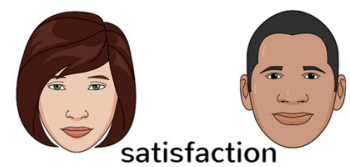

Fig. 3. Continuum-sorting hypothesis in antidepressant drug's perspective - part 2 . Antidepressants may enhance synaptic remodeling and/or neuronal turnover, whose final net wiring product may promote better cognitive flexibility, then culminating in a better decision-making. The state of the art of antidepressants would promote resilience to behavioral consequences of stress exposure such as depression and posttraumatic stress disorder. Legends: blue neurons are unmodified by antidepressant treatment; red neurons were added; and transparent neurons were excluded with antidepressant treatment. (For interpretation of the references to colour in this figure legend, the reader is referred to the web version of this article.)
Repeated sertraline (SSRI) treatment prevented behavioral consequences of stress exposure and increased tPA brain levels (Tang et al., 2015). Nonetheless, decreased hippocampal tPA levels were also found after CUS exposure (Zhang et al., 2016). Moreover, p75R knockout mice were more active in the tail-suspension test (TST), considered an antidepressant-like behavior (Catts et al., 2008). Li and co-workers found that zileuton, a lipoxygenase inhibitor, attenuated LPS-induced depressive-like behaviors while reversing the increase of 5-lipoxygenase levels, microglia activation, increased pro-inflammatory cytokines and decreased ratio of PCREB/CREB and mBDNF/proBDNF in the hippocampus of mice. However, it remains unclear whether this change of $\mathrm{mBDNF} /$ proBDNF ratio after zileuton treatment or LPS challenge depended on increased BDNF and/or on decreased proBDNF levels (Li et al., 2017).

Several protocols that reduce depressive-like symptoms are able to modulate molecules that play a key role in proBDNF cleavage to mBDNF. To a greater extent than reducing immobility time in the FST and increasing sucrose preference of rats, enriched environment upregulated hippocampal levels of matrix metalloproteinase-9, which is important to convert proBDNF to mBDNF in the extracellular space (Cao et al., 2014). Voluntary physical activity decreased immobility time on either FST or TST and induced an increase in hippocampal p11 and tPA mRNA expression (Sartori et al., 2011). Similar to tPA, p11 takes part in the cleavage of proBDNF (Sartori et al., 2011). Furthermore, electroconvulsive shocks, which is the most effective antidepressant treatment, increased furin, prohormone convertase 1 (PC1) and tPA mRNA expression in rat hippocampus (Segawa et al., 2013). The activity of tPA was also found increased in hippocampus (Segawa et al., 2013). Considering all the three studies described here, an increase of hippocampal mBDNF levels was observed, but an increase in the proBDNF levels was only observed in response electroconvulsive protocols. However, another study also using an electroconvulsive protocol found proBDNF levels unchanged in dorsal and ventral hippocampus (Enomoto et al., 2017).

Considering that mBDNF expression is completely tied to BDNF precursor (proBDNF), some questions may arise: 1) Would it be the case that increased mBDNF levels is secondary to proBDNF decrease regarding antidepressant effectivity?; 2) How independent is the opposite functional role of proBDNF and mBDNF from each other?; 3) Could it be that the ratio of proBDNF/mBDNF provides a phenotypic index for diagnosis of depression or treatment effectiveness?; and 4) Would pharmacological treatment targeting proBDNF/p75 signaling be a better clinical approach compared to pharmacological treatment targeting mBDNF/TrkB signaling?

Some studies have already provided some insights into these issues. In fact, by considering clinical research or post mortem studies, combination analysis of tPA, mBDNF, TrkB, proBDNF, and p75 levels yielded excellent diagnostic performance (Jiang et al., 2017). Furthermore, mBDNF is found at lower levels in blood, apparently because proBDNF is found at higher levels (Jiang et al., 2017; Yang et al., 2017a). However, a causal link or a hierarchical function between $\mathrm{BDNF}$, proBDNF, and the propeptide is still overshadowed regarding depressive disorder.

Since the increase in mBDNF levels is probably accompanied by BDNF-propeptide production, we cannot completely discard BDNF/p75 signaling as an important pathway to resolution of depressive symptoms. It is still possible that an elusive physiological effect from BDNFpropeptide acting on $\mathrm{p} 75 \mathrm{R}$, added to the mBDNF acting on TrkB receptor, could be important to counteract depressive symptoms. Depression, as a maladaptive consequence of recurrent and enduring stress exposure, involves a paramount negative effect of stress on neuronal plasticity in patients. Corroborating this proposal, depressive symptoms do not include only anhedonia, but motivational salience is also lost for pleasure, or even sadness. Such a cluster of symptoms could be the consequence of a globally bunted network. Therefore, it is plausible to consider that in depressive patients, neuronal turnover is a minor necessity to antidepressant effectiveness, since the greater requirement to reverse symptoms favors neuroplasticity positively.

\section{Concluding remarks}

The purpose of this work was to devise a link between mBDNF and proBDNF/propeptide for antidepressant therapeutic effect in reversing the deleterious effects of stress. Despite mBDNF and proBDNF/ 
propeptide usually having opposite functional roles on physiological effects, they might also present complimentary roles to modulate homeostatic balance between network efficacy and energy expenditure behind the best coping strategies to environmental contingences. Although the predominant observable effect from antidepressant treatment is a positive modulation of neuroplasticity, "recycling," either by synaptic remodeling or neuronal turnover, would be the core global effect of antidepressants (see Fig. 3). Importantly, the "continuum-sorting hypothesis" is an assumption around very scarce data regarding proBDNF and the propeptide functional role in the mechanism of action of antidepressants, even considering the neurobiology of the most studied psychiatric disorders, such as depression and PTSD. Therefore, the molecular mechanism in background of the hypothesis, respecting how mBDNF and/or proBDNF/propeptide would be balanced is still not crystal clear. Thus, more studies and data availability are required to aid the resolution of the issues raised here. In this sense, this review provides value in suggesting a new mechanism behind antidepressant action and intends to encourage research in this area.

Future analysis of the involvement of proBDNF and propeptide on neuronal plasticity and antidepressant response, especially during stressful situations, could shed light on this theme and reveal important mechanisms related to the behavioral effects obtained following antidepressant treatment. Another important consequence of this hypothesis refers to evidence-based theoretical constructs supporting the interplay between pharmacological treatment and cognitive therapy. Since antidepressant treatment boosts the capacity of neuronal remodeling, behavioral cognitive therapy would set the environmental selection of the appropriate neuronal contacts.

Therefore, beyond good and evil, the "continuum-sorting hypothesis" proposes that both $\mathrm{mBDNF} /$ proBDNF and/or mBDNF/propeptide signaling act as a fine-tuner of antidepressant-dependent neuroplasticity.

\section{Conflict of interest}

The authors declare no conflict of interest.

\section{Source of funding}

CNPq, FAPESP and ERC.

\section{Conflict of interest}

None.

\section{Acknowledgements}

The present research was supported by grants from FAPESP and CNPq (Brazil) and ERC (Finland).

\section{References}

Adachi, M., et al., 2008. Selective loss of brain-derived neurotrophic factor in the dentate gyrus attenuates antidepressant efficacy. Biol. Psychiatry 63 (7), 642-649.

Aid, T., et al., 2007. Mouse and rat BDNF gene structure and expression revisited. J. Neurosci. Res. 85 (3), 525-535.

Aimone, J.B., Gage, F.H., 2011. Modeling new neuron function: a history of using computational neuroscience to study adult neurogenesis. Eur. J. Neurosci. 33 (6), 1160-1169.

Aimone, J.B., Wiles, J., Gage, F.H., 2006. Potential role for adult neurogenesis in the encoding of time in new memories. Nat. Neurosci. 9 (6), 723-727.

Aimone, J.B., Deng, W., Gage, F.H., 2011. Resolving new memories: a critical look at the dentate gyrus, adult neurogenesis, and pattern separation. Neuron 70 (4), 589-596.

Alderson, R.F., et al., 1990. Brain-derived neurotrophic factor increases survival and differentiated functions of rat septal cholinergic neurons in culture. Neuron 5 (3), 297-306.

Aloyz, R.S., et al., 1998. p53 is essential for developmental neuron death as regulated by the TrkA and p75 neurotrophin receptors. J. Cell. Biol. 143 (6), 1691-1703.
An, J.J., et al., 2008. Distinct role of long 3' UTR BDNF mRNA in spine morphology and synaptic plasticity in hippocampal neurons. Cell 134 (1), 175-187.

Anacker, C., Hen, R., 2017. Adult hippocampal neurogenesis and cognitive flexibility linking memory and mood. Nat. Rev. Neurosci. 18 (6), 335-346.

Anastasia, A., et al., 2013. Val66Met polymorphism of BDNF alters prodomain structure to induce neuronal growth cone retraction. Nat. Commun. 4, 2490.

Araque, A., et al., 2014. Gliotransmitters travel in time and space. Neuron 81 (4), $728-739$.

Bai, Y.Y., et al., 2016. ProBDNF signaling regulates depression-like behaviors in rodents under chronic stress. Neuropsychopharmacology 41 (12), 2882-2892.

Baj, G., et al., 2011. Spatial segregation of BDNF transcripts enables BDNF to differentially shape distinct dendritic compartments. Proc. Natl. Acad. Sci. U. S. A. 108 (40), 16813-16818.

Baj, G., et al., 2012. Physical exercise and antidepressants enhance BDNF targeting in hippocampal CA3 dendrites: further evidence of a spatial code for BDNF splice variants. Neuropsychopharmacology 37 (7), 1600-1611.

Bamji, S.X., et al., 1998. The p75 neurotrophin receptor mediates neuronal apoptosis and is essential for naturally occurring sympathetic neuron death. J. Cell. Biol. 140 (4), 911-923.

Barnes, P., Thomas, K.L., 2008. Proteolysis of proBDNF is a key regulator in the formation of memory. PLoS One 3 (9), e3248.

Bath, K.G., et al., 2012. BDNF Val66Met impairs fluoxetine-induced enhancement of adult hippocampus plasticity. Neuropsychopharmacology 37 (5), 1297-1304.

Becker, E.B., et al., 2004. Characterization of the c-Jun kinase-BimEL signaling pathway in neuronal apoptosis. J. Neurosci. 24 (40), 8762-8770.

Bekinschtein, P., et al., 2013. BDNF in the dentate gyrus is required for consolidation of "pattern-separated" memories. Cell Rep. 5 (3), 759-768.

Bekinschtein, P., et al., 2014. Brain-derived neurotrophic factor interacts with adult-born immature cells in the dentate gyrus during consolidation of overlapping memories. Hippocampus 24 (8), 905-911.

Besnard, A., Sahay, A., 2016. Adult hippocampal neurogenesis, fear generalization, and stress. Neuropsychopharmacology 41 (1), 24-44.

Bessa, J.M., et al., 2009. The mood-improving actions of antidepressants do not depend on neurogenesis but are associated with neuronal remodeling. Mol. Psychiatry 14 (8), 764-773 739.

Bevilaqua, L.R., et al., 2007. Inhibition of c-Jun N-terminal kinase in the CA1 region of the dorsal hippocampus blocks extinction of inhibitory avoidance memory. Behav. Pharmacol. 18 (5-6), 483-489.

Bhakar, A.L., et al., 2003. Apoptosis induced by p75NTR overexpression requires Jun kinase-dependent phosphorylation of Bad. J. Neurosci. 23 (36), 11373-11381.

Bliss, T.V., Collingridge, G.L., 1993. A synaptic model of memory: long-term potentiation in the hippocampus. Nature 361 (6407), 31-39.

Brigman, J.L., et al., 2010. Loss of GluN2B-containing NMDA receptors in CA1 hippocampus and cortex impairs long-term depression, reduces dendritic spine density, and disrupts learning. J. Neurosci. 30 (13), 4590-4600.

Brunson, K.L., et al., 2005. Mechanisms of late-onset cognitive decline after early-life stress. J. Neurosci. 25 (41), 9328-9338.

Cao, W., et al., 2014. Early enriched environment induces an increased conversion of proBDNF to BDNF in the adult rat's hippocampus. Behav. Brain Res. 265, 76-83.

Capsoni, S., et al., 1999. Differential regulation of brain-derived neurotrophic factor messenger RNA cellular expression in the adult rat visual cortex. Neuroscience 93 (3), 1033-1040.

Caputo, V., et al., 2011. Brain derived neurotrophic factor (BDNF) expression is regulated by microRNAs miR-26a and miR-26b allele-specific binding. PLoS One 6 (12), e28656.

Castren, E., 2005. Is mood chemistry? Nat. Rev. Neurosci. 6 (3), 241-246.

Castren, E., 2013. Neuronal network plasticity and recovery from depression. JAMA Psychiatry 70 (9), 983-989.

Castren, E., Antila, H., 2017. Neuronal plasticity and neurotrophic factors in drug responses. Mol. Psychiatry 22 (8), 1085-1095.

Castren, E., Kojima, M., 2017. Brain-derived neurotrophic factor in mood disorders and antidepressant treatments. Neurobiol. Dis. 97 (Pt B), 119-126.

Castren, E., Rantamaki, T., 2010. The role of BDNF and its receptors in depression and antidepressant drug action: reactivation of developmental plasticity. Dev. Neurobiol. 70 (5), 289-297.

Castren, E., et al., 1993. The induction of LTP increases BDNF and NGF mRNA but decreases NT-3 mRNA in the dentate gyrus. Neuroreport 4 (7), 895-898.

Catts, V.S., et al., 2008. The p75 neurotrophin receptor regulates hippocampal neurogenesis and related behaviours. Eur. J. Neurosci. 28 (5), 883-892.

Chen, B., et al., 2001a. Increased hippocampal BDNF immunoreactivity in subjects treated with antidepressant medication. Biol. Psychiatry 50 (4), 260-265.

Chen, A.C., et al., 2001b. Expression of the cAMP response element binding protein (CREB) in hippocampus produces an antidepressant effect. Biol. Psychiatry 49 (9), $753-762$.

Chen, Z.Y., et al., 2005. Sortilin controls intracellular sorting of brain-derived neurotrophic factor to the regulated secretory pathway. J. Neurosci. 25 (26), 6156-6166.

Chen, Z.Y., et al., 2006. Genetic variant BDNF (Val66Met) polymorphism alters anxietyrelated behavior. Science 314 (5796), 140-143.

Chen, J., et al., 2016. proBDNF attenuates hippocampal neurogenesis and induces learning and memory deficits in aged mice. Neurotox. Res. 29 (1), 47-53.

Chiaruttini, C., et al., 2008. BDNF mRNA splice variants display activity-dependent targeting to distinct hippocampal laminae. Mol. Cell. Neurosci. 37 (1), 11-19.

Coffey, E.T., 2014. Nuclear and cytosolic JNK signalling in neurons. Nat. Rev. Neurosci. 15 (5), 285-299.

Condorelli, D.F., et al., 1994. Expression of neurotrophins and their receptors in primary astroglial cultures: induction by cyclic AMP-elevating agents. J. Neurochem. 63 (2), 
509-516.

Deng, W., et al., 2009. Adult-born hippocampal dentate granule cells undergoing maturation modulate learning and memory in the brain. J. Neurosci. 29 (43), $13532-13542$.

Dieni, S., et al., 2012. BDNF and its pro-peptide are stored in presynaptic dense core vesicles in brain neurons. J. Cell. Biol. 196 (6), 775-788.

Dong, Z., et al., 2013. Hippocampal long-term depression mediates spatial reversal learning in the Morris water maze. Neuropeptides (Oxford, United Kingdom) 64, 65-73.

Dowlatshahi, D., et al., 1998. Increased temporal cortex CREB concentrations and antidepressant treatment in major depression. Lancet 352 (9142), 1754-1755.

Duman, R.S., Monteggia, L.M., 2006. A neurotrophic model for stress-related mood disorders. Biol. Psychiatry 59 (12), 1116-1127.

Duman, R.S., et al., 2000. Neuronal plasticity and survival in mood disorders. Biol. Psychiatry 48 (8), 732-739.

Duman, C.H., et al., 2007. A role for MAP kinase signaling in behavioral models of depression and antidepressant treatment. Biol. Psychiatry 61 (5), 661-670.

Enomoto, S., et al., 2017. Activated brain-derived neurotrophic factor/TrkB signaling in rat dorsal and ventral hippocampi following 10-day electroconvulsive seizure treatment. Neurosci. Lett. 660, 45-50.

Eskandari, F., et al., 2005. Younger, premenopausal women with major depressive disorder have more abdominal fat and increased serum levels of prothrombotic factors: implications for greater cardiovascular risk. Metabolism 54 (7), 918-924.

Evans, S.F., et al., 2001. Neuronal brain-derived neurotrophic factor is synthesized in excess, with levels regulated by sortilin-mediated trafficking and lysosomal degradation. J. Biol. Chem. 286 (34), 29556-29567.

Fang, F., et al., 2017. Synuclein impairs trafficking and signaling of BDNF in a mouse model of Parkinson's disease. Sci. Rep. 7 (1), 3868.

Frade, J.M., Rodriguez-Tebar, A., Barde, Y.A., 1996. Induction of cell death by endogenous nerve growth factor through its p75 receptor. Nature 383 (6596), 166-168.

Friedman, W.J., 2000. Neurotrophins induce death of hippocampal neurons via the p75 receptor. J. Neurosci. 20 (17), 6340-6346.

Fuchs, S.Y., et al., 1998. MEKK1/JNK signaling stabilizes and activates p53. Proc. Natl. Acad. Sci. U. S. A. 95 (18), 10541-10546.

Gartner, A., et al., 2006. Hippocampal long-term potentiation is supported by presynaptic and postsynaptic tyrosine receptor kinase B-mediated phospholipase Cgamma signaling. J. Neurosci. 26 (13), 3496-3504.

Ge, Y., et al., 2010. Hippocampal long-term depression is required for the consolidation of spatial memory. Proc. Natl. Acad. Sci. U. S. A. 107 (38), 16697-16702 142.

Ghosh, A., Carnahan, J., Greenberg, M.E., 1994. Requirement for BDNF in activity-dependent survival of cortical neurons. Science 263 (5153), 1618-1623.

Gibon, J., Barker, P.A., 2017. Neurotrophins and proneurotrophins. Neuroscientist. http://dx.doi.org/10.1177/1073858417697037.

Gibon, J., Barker, P.A., Seguela, P., 2016. Opposing presynaptic roles of BDNF and ProBDNF in the regulation of persistent activity in the entorhinal cortex. Mol. Brain 9, 23.

Gooney, M., Lynch, M.A., 2001. Long-term potentiation in the dentate gyrus of the rat hippocampus is accompanied by brain-derived neurotrophic factor-induced activation of TrkB. J. Neurochem. 77 (5), 1198-1207.

Gottschalk, W.A., et al., 1999. Signaling mechanisms mediating BDNF modulation of synaptic plasticity in the hippocampus. Learn. Mem. 6 (3), 243-256.

Govindarajan, A., et al., 2006. Transgenic brain-derived neurotrophic factor expression causes both anxiogenic and antidepressant effects. Proc. Natl. Acad. Sci. U. S. A. 103 (35), 13208-13213.

Grutzendler, J., Kasthuri, N., Gan, W.B., 2002. Long-term dendritic spine stability in the adult cortex. Nature 420 (6917), 812-816.

Guo, J., et al., 2016. BDNF pro-peptide regulates dendritic spines via caspase-3. Cell Death Dis. 7, e2264.

Hajszan, T., MacLusky, N.J., Leranth, C., 2005. Short-term treatment with the antidepressant fluoxetine triggers pyramidal dendritic spine synapse formation in rat hippocampus. Eur. J. Neurosci. 21 (5), 1299-1303.

Halassa, M.M., Haydon, P.G., 2010. Integrated brain circuits: astrocytic networks modulate neuronal activity and behavior. Annu. Rev. Physiol. 72, 335-355.

Harrington, A.W., Kim, J.Y., Yoon, S.O., 2002. Activation of Rac GTPase by p75 is necessary for c-jun N-terminal kinase-mediated apoptosis. J. Neurosci. 22 (1), 156-166.

Hisaoka, K., et al., 2008. Antidepressants induce acute CREB phosphorylation and CREmediated gene expression in glial cells: a possible contribution to GDNF production. Brain Res. 1196, 53-58.

Hofer, M.M., Barde, Y.A., 1998. Brain-derived neurotrophic factor prevents neuronal death in vivo. Nature 331 (6153), 261-262.

Huang, E.J., Reichardt, L.F., 2001. Neurotrophins: roles in neuronal development and function. Annu, Rev, Neurosci. 24, 677-736.

Hung, H.C., Hsiao, Y.H., Gean, P.W., 2015. Sonic hedgehog signaling regulates amygdalar neurogenesis and extinction of fear memory. Eur. Neuropsychopharmacol. 25 (10), 1723-1732.

Hyman, C., et al., 1991. BDNF is a neurotrophic factor for dopaminergic neurons of the substantia nigra. Nature 350 (6315), 230-232.

Izquierdo, A., Wellman, C.L., Holmes, A., 2006. Brief uncontrollable stress causes dendritic retraction in infralimbic cortex and resistance to fear extinction in mice. $J$. Neurosci. 26 (21), 5733-5738.

Je, H.S., et al., 2012. Role of pro-brain-derived neurotrophic factor (proBDNF) to mature BDNF conversion in activity-dependent competition at developing neuromuscular synapses. Proc. Natl. Acad. Sci. U. S. A. 109 (39), 15924-15929.

Je, H.S., et al., 2013. ProBDNF and mature BDNF as punishment and reward signals for synapse elimination at mouse neuromuscular junctions. J. Neurosci. 33 (24), 9957-9962.
Ji, Y., et al., 2010. Acute and gradual increases in BDNF concentration elicit distinct signaling and functions in neurons. Nat. Neurosci. 13 (3), 302-309.

Jiang, L., et al., 2014. Fluoxetine pretreatment promotes neuronal survival and maturation after auditory fear conditioning in the rat amygdala. PLoS One 9 (2), e89147.

Jiang, H., et al., 2017. The serum protein levels of the tPA-BDNF pathway are implicated in depression and antidepressant treatment. Transl. Psychiatry 7 (4), e1079.

Johnson, J.E., et al., 1986. Brain-derived neurotrophic factor supports the survival of cultured rat retinal ganglion cells. J. Neurosci. 6 (10), 3031-3038.

Kalb, R., 2005. The protean actions of neurotrophins and their receptors on the life and death of neurons. Trends Neurosci. 28 (1), 5-11.

Karpova, N.N., et al., 2011. Fear erasure in mice requires synergy between antidepressant drugs and extinction training. Science 334 (6063), 1731-1734.

Kenchappa, R.S., et al., 2010. p75 neurotrophin receptor-mediated apoptosis in sympathetic neurons involves a biphasic activation of JNK and up-regulation of tumor necrosis factor-alpha-converting enzyme/ADAM17. J. Biol. Chem. 285 (26), 20358-20368.

Knusel, B., et al., 1991. Promotion of central cholinergic and dopaminergic neuron differentiation by brain-derived neurotrophic factor but not neurotrophin 3. Proc. Natl. Acad. Sci. U. S. A. 88 (3), 961-965.

Kobayashi, K., et al., 2010. Reversal of hippocampal neuronal maturation by serotonergic antidepressants. Proc. Natl. Acad. Sci. U. S. A. 107 (18), 8434-8439.

Koizumi, S., Fujishita, K., Inoue, K., 2005. Regulation of cell-to-cell communication mediated by astrocytic ATP in the CNS. Purinergic Signal. 1 (3), 211-217.

Koponen, E., et al., 2004a. Transgenic mice overexpressing the full-length neurotrophin receptor trkB exhibit increased activation of the trkB-PLCgamma pathway, reduced anxiety, and facilitated learning. Mol. Cell. Neurosci. 26 (1), 166-181.

Koponen, E., Lakso, M., Castren, E., 2004b. Overexpression of the full-length neurotrophin receptor trkB regulates the expression of plasticity-related genes in mouse brain. Brain Res. Mol. Brain Res. 130 (1-2), 81-94.

Koponen, E., et al., 2005. Enhanced BDNF signaling is associated with an antidepressantlike behavioral response and changes in brain monoamines. Cell. Mol. Neurobiol. 25 (6), 973-980.

Kraemer, B.R., Yoon, S.O., Carter, B.D., 2014. The biological functions and signaling mechanisms of the p75 neurotrophin receptor. Handb. Exp. Pharmacol. 220, 121-164.

Lau, A.G., et al., 2010. Distinct 3'UTRs differentially regulate activity-dependent translation of brain-derived neurotrophic factor (BDNF). Proc. Natl. Acad. Sci. U. S. A. 107 (36), 15945-15950.

Lee, R., et al., 2001. Regulation of cell survival by secreted proneurotrophins. Science 294 (5548), 1945-1948.

Li, Y., et al., 2008. TrkB regulates hippocampal neurogenesis and governs sensitivity to antidepressive treatment. Neuron 59 (3), 399-412.

Li, D.D., et al., 2017. Antidepressant-like effect of zileuton is accompanied by hippocampal neuroinflammation reduction and CREB/BDNF upregulation in lipopolysaccharide-challenged mice. J. Affect Disord. 227, 672-680.

Lichtman, J.W., Colman, H., 2000. Synapse elimination and indelible memory. Neuron 25 (2), 269-278.

Lindholm, J.S., Castren, E., 2014. Mice with altered BDNF signaling as models for mood disorders and antidepressant effects. Front. Behav. Neurosci. 8, 143.

Linggi, M.S., et al., 2005. Neurotrophin receptor interacting factor (NRIF) is an essential mediator of apoptotic signaling by the p75 neurotrophin receptor. J. Biol. Chem. 280 (14), 13801-13808.

Liston, C., et al., 2006. Stress-induced alterations in prefrontal cortical dendritic morphology predict selective impairments in perceptual attentional set-shifting. J. Neurosci. 26 (30), 7870-7874.

Lu, B., 2003a. Pro-region of neurotrophins: role in synaptic modulation. Neuron 39 (5), $735-738$.

Lu, B., 2003b. BDNF and activity-dependent synaptic modulation. Learn. Mem. 10 (2), 86-98.

Lu, B., Pang, P.T., Woo, N.H., 2005. The yin and yang of neurotrophin action. Nat. Rev. Neurosci. 6 (8), 603-614.

Ma, B., et al., 2010. Localization of BDNF mRNA with the Huntington's disease protein in rat brain. Mol. Neurodegener. 5, 22

Magarinos, A.M., Verdugo, J.M., McEwen, B.S., 1997. Chronic stress alters synaptic terminal structure in hippocampus. Proc. Natl. Acad. Sci. U. S. A. 94 (25), 14002-14008.

Manahan-Vaughan, D., Braunewell, K.H., 1999. Novelty acquisition is associated with induction of hippocampal long-term depression. Proc. Natl. Acad. Sci. U. S. A. 96 (15), 8739-8744.

Maren, S., Phan, K.L., Liberzon, I., 2013. The contextual brain: implications for fear conditioning, extinction and psychopathology. Nat. Rev. Neurosci. 14 (6), 417-428.

Marmigere, F., et al., 2003. Rapid induction of BDNF expression in the hippocampus during immobilization stress challenge in adult rats. Hippocampus 13 (5), 646-655.

Martinowich, K., et al., 2012. Roles of p75(NTR), long-term depression, and cholinergic transmission in anxiety and acute stress coping. Biol. Psychiatry 71 (1), 75-83.

Maya Vetencourt, J.F., et al., 2008. The antidepressant fluoxetine restores plasticity in the adult visual cortex. Science 320 (5874), 385-388.

Maya Vetencourt, J.F., et al., 2011. Serotonin triggers a transient epigenetic mechanism that reinstates adult visual cortex plasticity in rats. Eur. J. Neurosci. 33 (1), 49-57.

McAvoy, K., Besnard, A., Sahay, A., 2015. Adult hippocampal neurogenesis and pattern separation in DG: a role for feedback inhibition in modulating sparseness to govern population-based coding. Front. Syst. Neurosci. 9, 120.

Meeker, R.B., Williams, K.S., 2015. The p75 neurotrophin receptor: at the crossroad of neural repair and death. Neural Regen. Res. 10 (5), 721-725.

Metsis, M., et al., 1993. Differential usage of multiple brain-derived neurotrophic factor promoters in the rat brain following neuronal activation. Proc. Natl. Acad. Sci. U. S. 
A. 90 (19), 8802-8806.

Mizui, T., et al., 2015. BDNF pro-peptide actions facilitate hippocampal LTD and are altered by the common BDNF polymorphism Val66Met. Proc. Natl. Acad. Sci. U. S. A. 112 (23), E3067-74.

Monteggia, L.M., et al., 2004. Essential role of brain-derived neurotrophic factor in adult hippocampal function. Proc. Natl. Acad. Sci. U. S. A. 101 (29), 10827-10832.

Monteggia, L.M., et al., 2007. Brain-derived neurotrophic factor conditional knockouts show gender differences in depression-related behaviors. Biol. Psychiatry 61 (2), 187-197.

Nacher, J., et al., 2004. Chronic restraint stress and chronic corticosterone treatment modulate differentially the expression of molecules related to structural plasticity in the adult rat piriform cortex. Neuroscience 126 (2), 503-509.

Nagappan, G., et al., 2009. Control of extracellular cleavage of ProBDNF by high frequency neuronal activity. Proc. Natl. Acad. Sci. U. S. A. 106 (4), 1267-1272.

Nair, A., et al., 2007. Stressor-specific regulation of distinct brain-derived neurotrophic factor transcripts and cyclic AMP response element-binding protein expression in the postnatal and adult rat hippocampus. Neuropsychopharmacology 32 (7), 1504-1519.

Nakagawa, S., et al., 2002a. Localization of phosphorylated cAMP response elementbinding protein in immature neurons of adult hippocampus. J. Neurosci. 22 (22), 9868-9876.

Nakagawa, S., et al., 2002b. Regulation of neurogenesis in adult mouse hippocampus by cAMP and the cAMP response element-binding protein. J. Neurosci. 22 (9), 3673-3682.

Nibuya, M., Morinobu, S., Duman, R.S., 1995. Regulation of BDNF and trkB mRNA in rat brain by chronic electroconvulsive seizure and antidepressant drug treatments. J. Neurosci. 15 (11), 7539-7547.

Nibuya, M., Nestler, E.J., Duman, R.S., 1996. Chronic antidepressant administration increases the expression of cAMP response element binding protein (CREB) in rat hippocampus. J. Neurosci. 16 (7), 2365-2372.

Nykjaer, A., Willnow, T.E., Petersen, C.M., 2005. p75NTR-live or let die. Curr. Opin. Neurobiol. 15 (1), 49-57.

O'Leary, O.F., Wu, X., Castren, E., 2009. Chronic fluoxetine treatment increases expression of synaptic proteins in the hippocampus of the ovariectomized rat: role of BDNF signalling. Psychoneuroendocrinology 34 (3), 367-381.

Oe, S., Yoneda, Y., 2010. Cytoplasmic polyadenylation element-like sequences are involved in dendritic targeting of BDNF mRNA in hippocampal neurons. FEBS Lett. 584 (15), 3424-3430.

Oleinik, N.V., Krupenko, N.I., Krupenko, S.A., 2007. Cooperation between JNK1 and JNK2 in activation of p53 apoptotic pathway. Oncogene 26 (51), 7222-7230.

Orefice, L.L., et al., 2013. Distinct roles for somatically and dendritically synthesized brain-derived neurotrophic factor in morphogenesis of dendritic spines. J. Neurosci. 33 (28), 11618-11632.

Orefice, L.L., et al., 2016. Control of spine maturation and pruning through proBDNF synthesized and released in dendrites. Mol. Cell. Neurosci. 71, 66-79.

Pan, Y.W., et al., 2012. Inhibition of adult neurogenesis by inducible and targeted deletion of ERK5 mitogen-activated protein kinase specifically in adult neurogenic regions impairs contextual fear extinction and remote fear memory. J. Neurosci. 32 (19), 6444-6455.

Pang, P.T., et al., 2004. Cleavage of proBDNF by tPA/plasmin is essential for long-term hippocampal plasticity. Science 306 (5695), 487-491.

Panja, D., et al., 2014. Two-stage translational control of dentate gyrus LTP consolidation is mediated by sustained BDNF-TrkB signaling to MNK. Cell. Rep. 9 (4), 1430-1445.

Park, H., Poo, M.M., 2013. Neurotrophin regulation of neural circuit development and function. Nat. Rev. Neurosci. 14 (1), 7-23.

Pattabiraman, P.P., et al., 2005. Neuronal activity regulates the developmental expression and subcellular localization of cortical BDNF mRNA isoforms in vivo. Mol. Cell. Neurosci. 28 (3), 556-570.

Patterson, S.L., et al., 1996. Recombinant BDNF rescues deficits in basal synaptic trans mission and hippocampal LTP in BDNF knockout mice. Neuron 16 (6), 1137-1145.

Pattwell, S.S., et al., 2012. The BDNF Val66Met polymorphism impairs synaptic transmission and plasticity in the infralimbic medial prefrontal cortex. J. Neurosci. 32 (7), $2410-2421$.

Perera, T.D., et al., 2011. Necessity of hippocampal neurogenesis for the therapeutic action of antidepressants in adult nonhuman primates. PLoS One 6 (4), e17600.

Pham, K., et al., 2003. Repeated restraint stress suppresses neurogenesis and induces biphasic PSA-NCAM expression in the adult rat dentate gyrus. Eur. J. Neurosci. 17 (4), 879-886.

Popova, D., et al., 2014. Combination of fluoxetine and extinction treatments forms a unique synaptic protein profile that correlates with long-term fear reduction in adult mice. Eur. Neuropsychopharmacol. 24 (7), 1162-1174.

Post, R.M., 1992. Transduction of psychosocial stress into the neurobiology of recurrent affective disorder. Am. J. Psychiatry 149 (8), 999-1010.

Pozo, K., Goda, Y., 2010. Unraveling mechanisms of homeostatic synaptic plasticity. Neuron 66 (3), 337-351.

Pozzo-Miller, L.D., et al., 1999. Impairments in high-frequency transmission, synaptic vesicle docking, and synaptic protein distribution in the hippocampus of BDNF knockout mice. J. Neurosci. 19 (12), 4972-4983.

Pruunsild, P., et al., 2007. Dissecting the human BDNF locus: bidirectional transcription, complex splicing, and multiple promoters. Genome Res. 90 (3), 397-406.

Qi, X., et al., 2008. Fluoxetine increases the activity of the ERK-CREB signal system and alleviates the depressive-like behavior in rats exposed to chronic forced swim stress. Neurobiol. Dis. 31 (2), 278-285.

Qiao, H., et al., 2014. Progressive alterations of hippocampal CA3-CA1 synapses in an animal model of depression. Behav. Brain Res. 275, 191-200.

Qiao, H., et al., 2017. Role of proBDNF and BDNF in dendritic spine plasticity and depressive-like behaviors induced by an animal model of depression. Brain Res. 1663,
29-37.

Radley, J.J., et al., 2006. Repeated stress induces dendritic spine loss in the rat medial prefrontal cortex. Cereb. Cortex 16 (3), 313-320.

Radley, J.J., et al., 2008. Repeated stress alters dendritic spine morphology in the rat medial prefrontal cortex. J. Comp. Neurol. 507 (1), 1141-1150.

Raju, C.S., et al., 2011. In neurons, activity-dependent association of dendritically transported mRNA transcripts with the transacting factor CBF-A is mediated by A2RE/RTS elements. Mol. Biol. Cell. 22 (11), 1864-1877.

Rantamaki, T., Yalcin, I., 2016. Antidepressant drug action-From rapid changes on network function to network rewiring. Prog. Neuropsychopharmacol. Biol. Psychiatry 64, 285-292.

Rantamaki, T., et al., 2007. Pharmacologically diverse antidepressants rapidly activate brain-derived neurotrophic factor receptor TrkB and induce phospholipase-Cgamma signaling pathways in mouse brain. Neuropsychopharmacology 32 (10), 2152-2162.

Rantamaki, T., et al., 2011. Antidepressant drugs transactivate TrkB neurotrophin receptors in the adult rodent brain independently of BDNF and monoamine transporter blockade. PLoS One 6 (6), e20567.

Ren, J., et al., 2013. The effects of intra-hippocampal microinfusion of D-cycloserine on fear extinction, and the expression of NMDA receptor subunit NR2B and neurogenesis in the hippocampus in rats. Prog. Neuropsychopharmacol. Biol. Psychiatry 44, 257-264.

Righi, M., Tongiorgi, E., Cattaneo, A., 2000. Brain-derived neurotrophic factor (BDNF) induces dendritic targeting of BDNF and tyrosine kinase B mRNAs in hippocampal neurons through a phosphatidylinositol-3 kinase-dependent pathway. J. Neurosci. 20 (9), 3165-3174.

Rinaldi, A., et al., 2010. Stress induces region specific alterations in microRNAs expression in mice. Behav. Brain Res. 208 (1), 265-269.

Roceri, M., et al., 2002. Early maternal deprivation reduces the expression of BDNF and NMDA receptor subunits in rat hippocampus. Mol. Psychiatry 7 (6), 609-616.

Ruan, C.S., et al., 2016. Mice with Sort1 deficiency display normal cognition but elevated anxiety-like behavior. Exp. Neurol. 281, 99-108.

Russo-Neustadt, A.A., et al., 2000. Physical activity and antidepressant treatment potentiate the expression of specific brain-derived neurotrophic factor transcripts in the rat hippocampus. Neuroscience 101 (2), 305-312.

Saarelainen, T., et al., 2003. Activation of the TrkB neurotrophin receptor is induced by antidepressant drugs and is required for antidepressant-induced behavioral effects. J. Neurosci. 23 (1), 349-357.

Sairanen, M., et al., 2005. Brain-derived neurotrophic factor and antidepressant drugs have different but coordinated effects on neuronal turnover, proliferation, and survival in the adult dentate gyrus. J. Neurosci. 25 (5), 1089-1094.

Sandi, C., 2004. Stress, cognitive impairment and cell adhesion molecules. Nat. Rev. Neurosci. 5 (12), 917-930.

Sandi, C., Loscertales, M., 1999. Opposite effects on NCAM expression in the rat frontal cortex induced by acute vs. chronic corticosterone treatments. Brain Res. 828 (1-2), 127-134.

Santarelli, L., et al., 2003. Requirement of hippocampal neurogenesis for the behavioral effects of antidepressants. Science 301 (5634), 805-809.

Sartori, C.R., et al., 2011. The antidepressive effect of the physical exercise correlates with increased levels of mature BDNF, and proBDNF proteolytic cleavage-related genes, p11 and tPA. Neuroscience 180, 9-18.

Sasi, M., et al., 2017. Neurobiology of local and intercellular BDNF signaling. Pflugers Arch. 469 (5-6), 593-610.

Schmidt, H.D., Duman, R.S., 2010. Peripheral BDNF produces antidepressant-like effects in cellular and behavioral models. Neuropsychopharmacology 35 (12), 2378-2391.

Sedel, F., Bechade, C., Triller, A., 1999. Nerve growth factor (NGF) induces motoneuron apoptosis in rat embryonic spinal cord in vitro. Eur. J. Neurosci. 11 (11), 3904-3912.

Segawa, M., et al., 2013. Electroconvulsive seizure, but not imipramine, rapidly up-regulates pro-BDNF and t-PA, leading to mature BDNF production, in the rat hippocampus. Int. J. Neuropsychopharmacol. 16 (2), 339-350.

Seki, T., Rutishauser, U., 1998. Removal of polysialic acid-neural cell adhesion molecule induces aberrant mossy fiber innervation and ectopic synaptogenesis in the hippocampus. J. Neurosci. 18 (10), 3757-3766.

Shirayama, Y., et al., 2002. Brain-derived neurotrophic factor produces antidepressant effects in behavioral models of depression. J. Neurosci. 22 (8), 3251-3261.

Shirayama, Y., et al., 2015. Alterations in brain-derived neurotrophic factor (BDNF) and its precursor proBDNF in the brain regions of a learned helplessness rat model and the antidepressant effects of a TrkB agonist and antagonist. Eur. Neuropsychopharmacol. 25 (12), 2449-2458.

Smirnova, L., et al., 2005. Regulation of miRNA expression during neural cell specification. Eur. J. Neurosci. 21 (6), 1469-1477.

Smith, M.A., et al., 1995. Stress and glucocorticoids affect the expression of brain-derived neurotrophic factor and neurotrophin-3 mRNAs in the hippocampus. J. Neurosci. 15 (3 Pt 1), 1768-1777.

Son, Y., et al., 2015. Cranial irradiation regulates CREB-BDNF signaling and variant BDNF transcript levels in the mouse hippocampus. Neurobiol. Learn. Mem. 121, 12-19.

Soppet, D., et al., 1991. The neurotrophic factors brain-derived neurotrophic factor and neurotrophin-3 are ligands for the trkB tyrosine kinase receptor. Cell 65 (5), 895-903.

Southwick, S.M., Vythilingam, M., Charney, D.S., 2005. The psychobiology of depression and resilience to stress: implications for prevention and treatment. Annu. Rev. Clin. Psychol. 1, 255-291.

Steward, O., Schuman, E.M., 2001. Protein synthesis at synaptic sites on dendrites. Annu. Rev. Neurosci. 24, 299-325.

Sweatt, J.D., 2001. The neuronal MAP kinase cascade: a biochemical signal integration system subserving synaptic plasticity and memory. J. Neurochem. 76 (1), 1-10.

Tabassum, A., Khwaja, F., Djakiew, D., 2003. The p75(NTR) tumor suppressor induces 
caspase-mediated apoptosis in bladder tumor cells. Int. J. Cancer 105 (1), 47-52.

Takano, K., et al., 2012. Imipramine induces brain-derived neurotrophic factor mRNA expression in cultured astrocytes. J. Pharmacol. Sci. 120 (3), 176-186.

Taliaz, D., et al., 2011. Resilience to chronic stress is mediated by hippocampal brainderived neurotrophic factor. J. Neurosci. 31 (12), 4475-4483.

Tanaka, J., et al., 2008. Protein synthesis and neurotrophin-dependent structural plasticity of single dendritic spines. Science 319 (5870), 1683-1687.

Tang, M., et al., 2015. Antidepressant-like effect of n-3 PUFAs in CUMS rats: role of tPA/ PAI-1 system. Physiol. Behav. 139, 210-215.

Tao, X., et al., 1998. Ca2 + influx regulates BDNF transcription by a CREB family transcription factor-dependent mechanism. Neuron 20 (4), 709-726.

Teng, H.K., et al., 2005. ProBDNF induces neuronal apoptosis via activation of a receptor complex of p75NTR and sortilin. J. Neurosci. 25 (22), 5455-5463.

Timmusk, T., et al., 1993. Multiple promoters direct tissue-specific expression of the rat BDNF gene. Neuron 10 (3), 475-489.

Timmusk, T., et al., 1994. Developmental regulation of brain-derived neurotrophic factor messenger RNAs transcribed from different promoters in the rat brain. Neuroscience 60 (2), 287-291.

Tolwani, R.J., et al., 2002. BDNF overexpression increases dendrite complexity in hippocampal dentate gyrus. Neuroscience 114 (3), 795-805.

Tongiorgi, E., 2008. Activity-dependent expression of brain-derived neurotrophic factor in dendrites: facts and open questions. Neurosci. Res. 61 (4), 335-346.

Tongiorgi, E., Righi, M., Cattaneo, A., 1997. Activity-dependent dendritic targeting of BDNF and TrkB mRNAs in hippocampal neurons. J. Neurosci. 17 (24), 9492-9505.

Tongiorgi, E., et al., 2004. Brain-derived neurotrophic factor mRNA and protein are targeted to discrete dendritic laminas by events that trigger epileptogenesis. J. Neurosci. 24 (30), 6842-6852.

Uegaki, K., et al., 2017. BDNF binds its pro-peptide with High affinity and the Common Val66Met polymorphism attenuates the interaction. Int. J. Mol. Sci. 18 (5).

Unsain, N., et al., 2008. Status epilepticus induces a TrkB to p75 neurotrophin receptor switch and increases brain-derived neurotrophic factor interaction with p75 neurotrophin receptor: an initial event in neuronal injury induction. Neuroscience 154 (3), 978-993.

VanElzakker, M.B., et al., 2014. From Pavlov to PTSD: the extinction of conditioned fear in rodents, humans, and anxiety disorders. Neurobiol. Learn. Mem. 113, 3-18.

Varea, E., et al., 2007. Chronic fluoxetine treatment increases the expression of PSANCAM in the medial prefrontal cortex. Neuropsychopharmacology 32 (4), 803-812.

Vicario, A., et al., 2015. Dendritic targeting of short and long 3' UTR BDNF mRNA is regulated by BDNF or NT-3 and distinct sets of RNA-binding proteins. Front. Mol. Neurosci. 8, 62 .

Vollmayr, B., Mahlstedt, M.M., Henn, F.A., 2007. Neurogenesis and depression: what animal models tell us about the link. Eur. Arch. Psychiatry Clin. Neurosci. 257 (5), 300-303.

Volosin, M., et al., 2008. Induction of proneurotrophins and activation of p75NTRmediated apoptosis via neurotrophin receptor-interacting factor in hippocampal neurons after seizures. J. Neurosci. 28 (39), 9870-9879.

Wang, J.W., et al., 2008. Chronic fluoxetine stimulates maturation and synaptic plasticity of adult-born hippocampal granule cells. J. Neurosci. 28 (6), 1374-1384.
Warner, C.H., et al., 2013. Identifying and managing posttraumatic stress disorder. Am. Fam. Phys. 88 (12), 827-834.

West, A.E., Pruunsild, P., Timmusk, T., 2014. Neurotrophins: transcription and translation. Handb. Exp. Pharmacol. 220, 67-100.

Winnubst, J., et al., 2015. Spontaneous Activity Drives Local Synaptic Plasticity In Vivo. Neuron 87 (2), 399-410.

Woo, N.H., et al., 2005. Activation of p75NTR by proBDNF facilitates hippocampal longterm depression. Nat. Neurosci. 8 (8), 1069-1077.

Wu, Y.C., et al., 2011. Dendritic trafficking of brain-derived neurotrophic factor mRNA: regulation by translin-dependent and -independent mechanisms. J. Neurochem. 116 (6), 1112-1121.

Xing, J., Ginty, D.D., Greenberg, M.E., 1996. Coupling of the RAS-MAPK pathway to gene activation by RSK2, a growth factor-regulated CREB kinase. Science 273 (5277), 959-963.

Yang, J., et al., 2009. Neuronal release of proBDNF. Nat. Neurosci. 12 (2), 113-115.

Yang, J., et al., 2014. proBDNF negatively regulates neuronal remodeling, synaptic transmission, and synaptic plasticity in hippocampus. Cell. Rep. 7 (3), 796-806.

Yang, B., et al., 2016. Regional differences in the expression of brain-derived neurotrophic factor (BDNF) pro-peptide, proBDNF and preproBDNF in the brain confer stress resilience. Eur. Arch. Psychiatry Clin. Neurosci. 266 (8), 765-769.

Yang, B., et al., 2017a. Altered expression of BDNF, BDNF pro-peptide and their precursor proBDNF in brain and liver tissues from psychiatric disorders: rethinking the brainliver axis. Transl. Psychiatry 7 (5), e1128.

Yang, C.R., et al., 2017b. Injection of Anti-proBDNF in Anterior Cingulate Cortex (ACC) Reverses Chronic Stress-Induced Adverse Mood Behaviors in Mice. Neurotox. Res. 31 (2), 298-308.

Yeiser, E.C., et al., 2004. Neurotrophin signaling through the p75 receptor is deficient in traf6-/- mice. J. Neurosci. 24 (46), 10521-10529.

Yoon, S.O., et al., 1998. Competitive signaling between TrkA and p75 nerve growth factor receptors determines cell survival. J. Neurosci. 18 (9), 3273-3281.

Yoshimura, R., et al., 2014. Serum proBDNF/BDNF and response to fluvoxamine in drugnaive first-episode major depressive disorder patients. Ann. Gen. Psychiatry 13, 19.

Yu, H., et al., 2009. Variant BDNF Val66Met polymorphism affects extinction of conditioned aversive memory. J. Neurosci. 29 (13), 4056-4064.

Yu, H., et al., 2012. Variant brain-derived neurotrophic factor Val66Met polymorphism alters vulnerability to stress and response to antidepressants. J. Neurosci. 32 (12), 4092-4101.

Zhang, H., Miller, R.H., Rutishauser, U., 1992. Polysialic acid is required for optimal growth of axons on a neuronal substrate. J. Neurosci. 12 (8), 3107-3114.

Zhang, F., et al., 2016. Propofol alleviates electroconvulsive shock-induced memory impairment by modulating proBDNF/mBDNF ratio in depressive rats. Brain Res. 1642, 43-50.

Zhou, L., et al., 2013. Upregulation of blood proBDNF and its receptors in major depression. J. Affect Disord. 150 (3), 776-784.

Zhu, Z., et al., 2016. Different effects of prenatal stress on ERK2/CREB/Bcl-2 expression in the hippocampus and the prefrontal cortex of adult offspring rats. Neuroreport 27 (8), 600-604. 